PROC. IMECHE-PART C - JOURNAL OF MECHANICAL ENGINEERING SCIENCE

Impact Factor: 1.015

eISSN: 20412983 | ISSN: 09544062

PUBLISHER: SAGE

Accepted February $22^{\text {nd }} 2018$

\title{
UNSTEADY REACTIVE MAGNETIC RADIATIVE MICROPOLAR FLOW, HEAT AND MASS TRANSFER FROM AN INCLINED PLATE WITH JOULE HEATING: A MODEL FOR MAGNETIC POLYMER PROCESSING
}

\author{
MD. Shamshuddin ${ }^{1 *}$, S.R. Mishra ${ }^{2}$, O. Anwar Bég ${ }^{3}$ and A. Kadir ${ }^{4}$ \\ ${ }^{1 *}$ Department of Mathematics, Vaagdevi College of Engineering, Warangal, Telangana, India. \\ ${ }^{2}$ Department of Mathematics, Siksha 'O'Anusandhan University, Khandagiri, Bhubaneswar, Odisha, India \\ ${ }^{3}$ Fluid Mechanics, Aeronautical and Mechanical Engineering, School of Computing, Science and Engineering, \\ Newton Building, The Crescent, Salford, M54WT, England, UK. \\ ${ }^{4}$ Materials, Corrosion and Structures, Aeronautical and Mechanical Engineering, School of Computing, Science \\ and Engineering, Newton Building, Salford, M54WT, England, UK. \\ *Corresponding author: shammaths@gmail.com, mdshamshuddin@vaagdevieng.ac.in \\ Contact No: +91-9866826099; Orchid Number: 0000-0002-2453-8492
}

\begin{abstract}
Magnetic polymer materials processing involves many multi-physical and chemical effects. Motivated by such applications, in the present work a theoretical analysis is conducted of combined heat and mass transfer in unsteady mixed convection flow of micropolar fluid over an oscillatory inclined porous plate in a homogenous porous medium with heat source, radiation absorption and Joule dissipation. A first order homogenous chemical reaction model is used. The transformed non-dimensional boundary value problem is solved using a perturbation method and Runge-Kutta fourth order numerical quadrature (shooting technique). The emerging parameters dictating the transport phenomena are shown to be the gyro-viscosity micropolar material parameter, magnetic field parameter, permeability of the porous medium, Prandtl number, Schmidt number, thermal Grashof number, species Grashof number, thermal radiation-conduction parameter, heat absorption parameter, radiation absorption parameter, Eckert number, chemical reaction parameter and Eringen coupling number (vortex viscosity ratio parameter). The impact of these parameters on linear velocity, microrotation (angular velocity), temperature and concentration are evaluated in detail. Results for skin friction coefficient, couple stress coefficient, Nusselt number and Sherwood number are also included. Couple stress is observed to be reduced with stronger magnetic field. Verification of solutions is achieved with earlier published analytical results.
\end{abstract}

KEY WORDS: Heat absorption; Radiation absorption; Joule dissipation; Micropolar fluid; Inclined plate.

\section{INTRODUCTION}

Heat absorption or generation effects arise in many complex thermal technologies featuring high temperature differences. These include fire and combustion processes, blast furnaces, nuclear reactor heat removal, material fabrication of powders, re-entry aero-thermodynamics, chemical engineering plant experiencing endothermic and/or exothermic chemical reactions and cooling of finned heat sinks [1]. Mathematical models of thermal 
convection flows with heat generation/absorption have therefore attracted significant attention in recent years. In these flows, temperature-dependent heat absorption may have a strong effect on heat and mass transfer characteristics of the processed materials which include polymers, ceramics, metals, slurries, plastics etc. [2]. Magnetic materials processing involves the application of static and/or alternating magnetic fields to control flow processes and modify material properties. This is achieved via the Lorentz magnetic body force. Numerous studies of magnetic transport phenomena have been communicated for a variety of complex fluids with many computational approaches. Khedr et al. [2] used a finite difference method to investigate hydromagnetic flow of micropolar fluids along a stretching permeable surface with heat generation of absorption effects. Pal and Biswas [3] studied the influence of heat sink on magneto-thermal radiative convective oscillatory flow of a micropolar fluid. Mishra et al. [4] investigated coupled free convection heat and mass transfer in magnetized micropolar flow with heat source. Srinivas Raju et al. [5] examined heat absorption effects both analytically (Laplace transform method) and numerically (finite element method) on unsteady magneto-convection flow over exponentially moving vertical plate with buoyancy effects. Other recent studies addressing micropolar flow with heat source/sink effects in different configurations include Alam et al. [6] (stretching/shrinking wedge), Mishra et al. [7] and Tripathy et al. [8]. Another type of heat absorption is the radiation absorption effect which can be invoked in certain materials processing systems. This effect must be considered in addition to thermal radiation heat flux which is usually simulated with algebraic flux models. A robust approach for analysing radiation absorption has been documented by Dombrovsky and Sazhin [9] who considered applications in vaporization process of $n$ decane, combustion processes in diesel engines and other high temperature systems [10]. Satyanarayana et al. [11] analysed Hall current and radiation absorption effects on hydromagnetic micropolar flow in a rotating system. Kundu et al. [12] examined radiation and thermal diffusion effects on MHD micropolar fluid flow in a rotating system. Harish Babu and Satyanarayana [13] reported on the influence of material permeability and radiation absorption on heat and mass transfer in magnetohydrodynamic micropolar convection from a moving vertical porous plate in a porous medium.

In magnetohydrodynamic flows, other phenomena may also arise including Hall currents, ion slip and Ohmic (Joule) dissipation. In most simulations of magnetic heat transfer, the Joule dissipation term is conventionally neglected on the premise that under normal conditions the Eckert number is small based on an order of magnitude analysis. Gebhart [14] presented one of the earliest and most definitive studies of viscous dissipation in natural convection. Rahman [15] studied the effects of viscous dissipation and Joule heating in convective flows of a micropolar fluid, observing that heat transfer rates are decreased with increasing Joule heating effect. Haque et al. 
[16] examined the steady magnetic natural convection heat transfer in micropolar fluid with Joule heating and viscous dissipation. Effects of viscous dissipation and heat source on an unsteady stretching sheet was examined by Reddy et al. [17]. Reddy and Gorla [18] reported Cattaneo-Christov heat flux and viscous dissipation effects on nonlinear convective stretching vertical surface for micropolar fluid. Reddy [19] extended the same study by incorporating Lorentz force effects.

Chemical reaction also arises in many industrial processes including reactive polymer flows in heterogeneous porous media, gel synthesis, corrosion phenomena in coated components, chemically-reactive vapour deposition boundary layers, catalytic combustion boundary layers and multi-stage reactions in metallurgical mass transfer and kinetics. Several investigations have considered reactive heat and mass transfer in external boundary layer flows for micropolar and other fluids. Sheri and Shamshuddin [20] have addressed the problem of coupled heat and mass transfer in magnetohydrodynamic micropolar flow with both viscous dissipation and chemical reaction effects. Rout et al. [21] employed a Runge-Kutta fourth order shooting technique to investigate the impact of chemical reaction on magnetohydrodynamic free convection flow in a micropolar fluid. Pal and Talukdar [22] used a perturbation technique to investigate time-dependent MHD mixed convection periodic flow, heat and mass transfer in micropolar fluid with chemical reaction in the presence of thermal radiation. Reddy [23] adopted perturbation technique to examine thermal radiation and chemical reaction effects on steady convective slip flow. Again, Reddy [24] also examined peristaltic flow in a porous medium with partial slip. Bég et al. [25] used a local similarity method to study free and forced convection reactive boundary layer flows with thermo-diffusion effects. Pal and Biswas [26] extended the same study by incorporating thermal radiation and viscous dissipation effects

In certain materials processing operations, buoyancy forces can be manipulated via inclination. The boundary layer flows adjacent to inclined plane surfaces (or indeed other geometries) are therefore of some relevance to elucidating the fluid dynamics of such applications. Rahman et al. [27] analysed heat transfer in micropolar fluid along an inclined permeable plate with variable fluid properties. Ajaz and Elangovan [28] investigated the action of alternating electric field with the effect of inclined magnetic field on the oscillatory flow of micropolar fluid. Other recent studies focused on micropolar fluid dynamics along inclined surface include the works by Aurangzaib et al. [29] and Srinivasacharya and Himabindu [30]. These studies all demonstrated the significant influence of inclination on thermofluid dynamic characteristics in micropolar fluids via modification of thermal and species buoyancy forces. Further studies of inclined plate multi-physical convection flows include Bég et al. [31] on magnetic-micropolar thin film flow, Bég et al. [32] on radiative gas convection, Bég et al. [33] on couple 
stress (polar) magnetic oscillatory flow, Rana et al. [34] on nanofluid convection in porous media and Rao et al. [35] on Casson boundary layer slip flows.

To the best of our knowledge, the present problem of magnetohydrodynamic oscillatory micropolar mixed convective heat and mass transfer from an inclined plate in porous media in the presence of heat source, radiation absorption, chemical reaction and Joule (Ohmic) dissipation has been unexplored. A theoretical model is developed for this flow scenario. Approximate pperturbation approximation form solutions presented by Pal and Biswas [26] provide a benchmark for the present numerical solution in which an efficient Runge-Kutta fourth order method with shooting quadrature is employed. The effects of various emerging thermo-physical parameters on the velocity, micro-rotation (angular) velocity, temperature and concentration profiles as well as on local skin friction coefficient, wall couple stress, Nusselt number and Sherwood number are visualized graphically. The study is relevant to multi-physical modelling of magnetic polymer processing [36].

\section{MATHEMATICAL FORMULATION}

The regime under investigation comprises an unsteady laminar magnetohydrodynamic free convective flow, heat and mass transfer in an electrically-conducting incompressible micropolar fluid, from a semi-infinite inclined permeable plate in the presence of heat absorption, radiation absorption, chemical reaction and Joule dissipation. The plate makes an angle $\alpha\left(0^{\circ} \leq \alpha \leq 90^{\circ}\right)$ to the vertical and is adjacent to a homogenous, isotropic, porous medium. The schematic model of physical problem is depicted in Fig. 1.

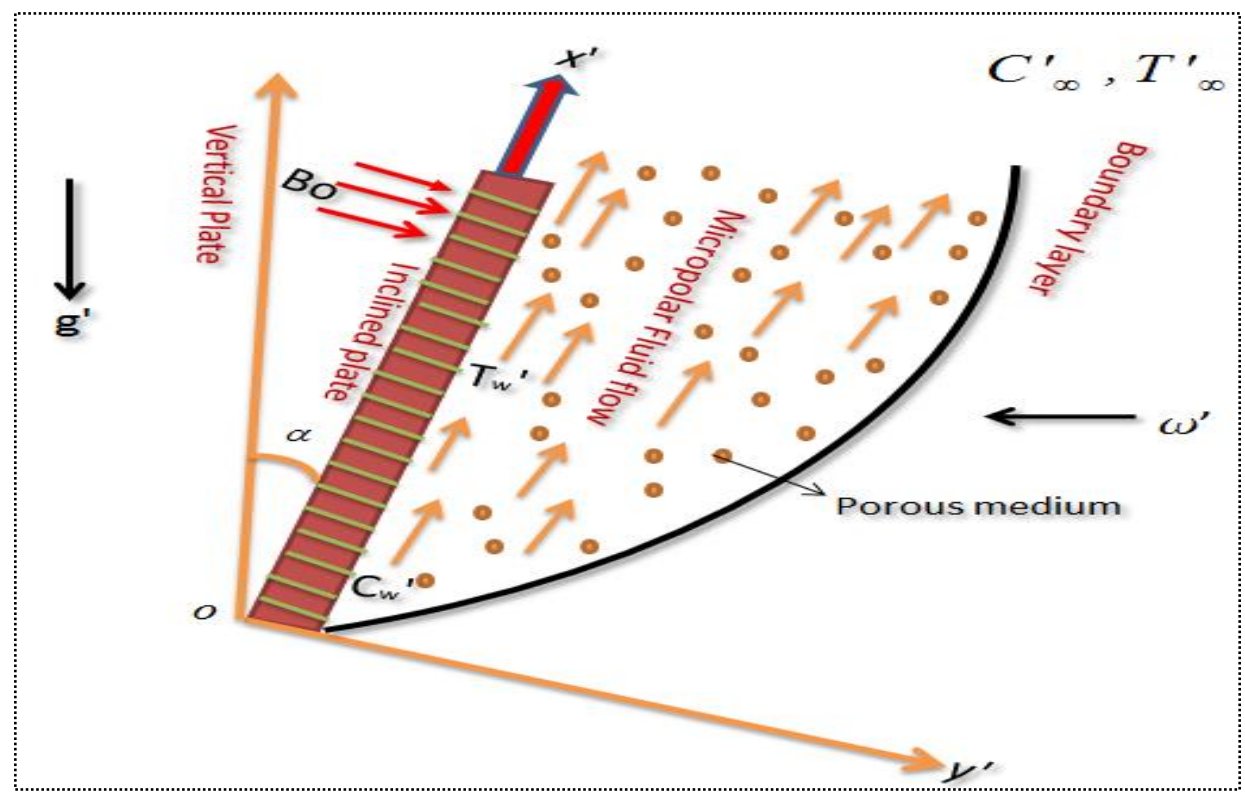

Fig .1: Schematic model of physical problem 
The Eringen [37] micropolar model is employed since it successfully captures microstructural characteristics of complex magnetic polymers i.e. it robustly simulates rotatory motions, gyration of fluid micro-elements. Micropolar fluids can support couple stresses, shear stresses, body couples and, also exhibit microrotational effects and inertia. They effectively model polymers as a dilute suspension of rigid macromolecules with individual motions. Micropolar theory therefore captures complex phenomena which are not realizable in other non-Newtonian models such as viscoelastic fluids and viscoplastic fluids. Another attraction of micropolar fluids is that the Navier-Stokes classical viscous flow model may be extracted as a special case. Extensive details of porous media micropolar flow simulation are provided in Bég et al. [38] and Bhargava et al. [39]. The inclination angles $0^{\circ}, 90^{\circ}$ and $0^{\circ} \leq \alpha \leq 90^{\circ}$ represents the vertical, horizontal and inclined plate respectively. Darcy's law is assumed and low Reynolds number flow (viscous-dominated regime). A uniform magnetic field of strength $B_{0}$ acts in a direction parallel to the $y^{\prime}$ axis which is perpendicular to the flow direction. Magnetic Reynolds number is very small so induced magnetic field is negligible in comparison to the applied magnetic field. It is also assumed that applied or polarized voltage is neglected so that no energy is added or extracted from the fluid by electrical means. The fluid is considered to be a gray, absorbing-emitting but non-scattering medium and the Rosseland approximation is used to describe the radiative heat flux. The radiative heat flux in the $x^{\prime}$ direction is considered negligible in comparison with that of $y^{\prime}$ direction. The magnetic micropolar fluid contains a species which is reactive and obeys first order chemical reaction. To simplify the formulation of the boundary conditions, we assumed the size of holes in the porous plate is significantly larger than the characteristic microscopic length scale of the micropolar fluid. It is further assumed that the plate is infinite in extent and hence all physical quantities depend only on $y^{\prime}$ and $t^{\prime}$. The equations governing the behaviour of an incompressible unsteady micropolar fluid in vectoral form are [37,40]:

\section{Conservation of mass:}

$\nabla . V=0$

\section{Conservation of momentum:}

$$
\rho\left(V_{t}+(V . \nabla) V\right)=-\nabla p+(\mu+k) \nabla^{2} V+k \nabla \times \Omega+\rho f
$$

\section{Conservation of angular momentum:}

$\rho j\left(\Omega_{t}+(V . \nabla) \Omega\right)=\left(\alpha_{0}+\beta_{0}+\gamma_{0}\right) \nabla(\nabla \Omega)-\gamma_{0} \nabla \times(\nabla \times \Omega)+k \nabla \times V-2 k \Omega+\rho l$ 


\section{Conservation of Energy:}

$$
\rho c_{p}\left(T_{t}+(V . \nabla) T\right)=\kappa \nabla^{2} T+\varphi+Q
$$

\section{Conservation of Species Concentration:}

$$
\left(C_{t}+(V . \nabla) C\right)=D \nabla^{2} C-k_{1}\left(C-C_{1}\right)
$$

Where $f$ is the body force per unit mass and $l$ is the body couple per unit mass, $V$ is the translational vector, $\Omega$ is the micro-rotation vector and $p$ is the pressure. $\alpha_{0}, \beta_{0}, \gamma_{0}$ and $k$ are the material constants for micropolar fluids. $\rho$ is the fluid density, $j$ is the micro-inertia, $\mu$ is the dynamic viscosity, $\kappa$ is the thermal conductivity, $T$ is the fluid temperature, $\varphi$ is the dissipation function and $c_{p}$ is the specific heat at constant pressure. Equations (1)- (5) represents conservation of mass, linear momentum, angular momentum, energy and species concentration respectively. We remark that for $k=\alpha_{0}=\beta_{0}=\gamma_{0}=0$ and vanishing $l$ and $f$, microrotation $\Omega$ becomes zero and equation (2) reduces to classical Navier-Stokes equations. Here microrotation does not affect the global motion since for $k=0$ the velocity and microrotation are not coupled.

By taking the aforesaid assumptions into consideration the governing boundary layer equations (see Mishra and Jena [41]) for unsteady convective oscillatory flow under Boussinesq's approximation are as follows:

$$
\begin{aligned}
& \frac{\partial v^{\prime}}{\partial y^{\prime}}=0 \\
& \frac{\partial u^{\prime}}{\partial t^{\prime}}+v^{\prime} \frac{\partial u^{\prime}}{\partial y^{\prime}}=\left(v+v_{r}\right) \frac{\partial^{2} u^{\prime}}{\partial y^{\prime 2}}+g \beta_{f}\left(T-T_{\infty}\right) \cos \alpha+g \beta_{c}\left(C-C_{\infty}\right) \cos \alpha-\frac{v}{K^{\prime}} u^{\prime}-\frac{v_{r}}{K^{\prime}} u^{\prime}-\frac{\sigma B_{0}^{2}}{\rho} u^{\prime}+2 v_{r} \frac{\partial \omega^{\prime}}{\partial y^{\prime}} \\
& \frac{\partial \omega^{\prime}}{\partial t^{\prime}}+v^{\prime} \frac{\partial \omega^{\prime}}{\partial y^{\prime}}=\frac{\gamma}{\rho j^{\prime}} \frac{\partial^{2} \omega^{\prime}}{\partial y^{\prime}} \\
& \frac{\partial T}{\partial t^{\prime}}+v^{\prime} \frac{\partial T}{\partial y^{\prime}}=\frac{\kappa}{\rho C_{p}} \frac{\partial^{2} T}{\partial y^{\prime 2}}-\frac{1}{\rho C_{p}} \frac{\partial q_{r}}{\partial y^{\prime}}-\frac{Q^{\prime}}{\rho C_{p}}\left(T-T_{\infty}\right)+\frac{Q_{1}^{\prime}}{\rho C_{p}}\left(C-C_{\infty}\right)+\frac{\mu}{\rho C_{p}}\left(\frac{\partial u^{\prime}}{\partial y^{\prime}}\right)^{2}+\frac{\sigma B_{0}^{2}}{\rho C_{p}} u^{\prime 2} \\
& \frac{\partial C}{\partial t^{\prime}}+v^{\prime} \frac{\partial C}{\partial y^{\prime}}=D \frac{\partial^{2} C}{\partial y^{\prime 2}}+K_{r}^{\prime}\left(C-C_{\infty}\right)
\end{aligned}
$$

Here $u^{\prime}$ and $v^{\prime}$ are velocity components along $x^{\prime}$ and $y^{\prime}$ axis respectively, $\omega^{\prime}$ is the microrotation component describes its relationship with the surface stress, $v$ is the kinematic viscosity, $v_{r}$ is the kinematic vortex viscosity, micro-rotation viscosity, $\rho$ is the density of magneto-micropolar fluid, $\beta f$ and $\beta c$ are coefficient of thermal expansion and concentration expansion, $K^{\prime}$ is permeability of porous medium, $\sigma$ is the electrical conductivity of 
the magneto-micropolar fluid, $\gamma$ is the material property (gyroscopic viscosity) of the magneto-micropolar fluid, $j^{\prime}$ is the micro inertia per unit mass, ${ }_{B_{0}}$ is the applied magnetic field strength, $g$ is the acceleration due to gravity, $T^{\prime}, T_{\infty}^{\prime}$ are temperature of fluid at the plate (wall) and far away from surface (free stream), $\kappa$ is thermal conductivity of the micropolar fluid-saturated porous medium. At constant pressure $p, C_{p}$ is the specific heat, $q_{r}$ is the heat flux, $Q^{\prime}$ is dimensional heat absorption, $Q_{1}^{\prime}$ is dimensional radiation absorption, $C^{\prime}, C_{\infty}^{\prime}$ are concentration of the solute and far away from surface, $D$ is the molecular diffusivity. It is assumed that the porous plate moves with a constant velocity in the longitudinal direction, the free stream velocity follows an exponentially increasing (or) decreasing value. The plate temperature and suction velocity vary exponentially with time and since there is change in the concentration of species (i.e. solutal), buoyancy effects arise and equation (7) and equation (10) are coupled. Under these assumptions following spatial and temporal boundary conditions are:

$$
\begin{aligned}
& t^{\prime} \leq 0 \quad\left\{u^{\prime}=0, \omega^{\prime}=0, T=T_{\infty}, C=C_{\infty} \quad \text { for all } y^{\prime} \geq 0\right.
\end{aligned}
$$

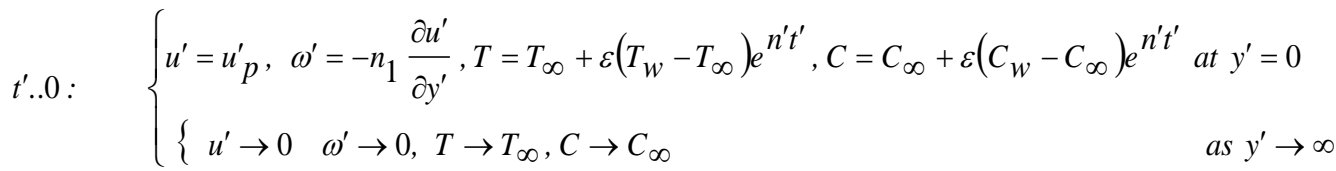

Where $n^{\prime}$ is a scalar constant, and $\varepsilon$ is a small quantity, the micro-rotation component in boundary condition i.e. $\omega^{\prime}=-n_{1}\left(\partial u^{\prime} / \partial y^{\prime}\right)$, describes its relationship with surface stresses. The parameter $n_{1}$ assumes values between 0 and 1 that quantifies the relationship between the micro-gyration vector to the shear stress. When $n_{1}=0$ this corresponds to the case where the particle density is sufficiently large so that microelements close to the wall are not able to rotate [42]. When $n_{1}=0.5$ indicates weak concentration and disappearance of anti-symmetric part of stress tensor as elaborated by Ahmadi [43]. When $n_{1}=1.0$ represents turbulent boundary layer flows as described by Peddieson [44] and Stokes [45]. However, when $n_{1}=0.5$ or $n_{1}=1.0$ this case tends to accelerate the flow $[44,45]$. Integrating the mass conservation (continuity) equation (1) for variable suction velocity normal to the plate which is taken, following Pal and Biswas [3] as:

$$
v^{\prime}=-V_{0}\left(1+\varepsilon A e^{n^{\prime} t^{\prime}}\right)
$$

Where $A$ is real constant such that $\varepsilon A<<1$ and $V_{0}$ is the normal velocity at the plate, negative sign indicates the suction velocity is directed towards the plate. Following Rosseland's approximation, the radiative heat flux term is given by: 
$q_{r}=\frac{-4 \bar{\sigma}}{3 \bar{k}}\left(\frac{\partial T^{4}}{\partial y^{\prime}}\right)$

Here $\bar{\sigma}$ and $\bar{k}$ are the Stefan-Boltzmann constant and mean absorption coefficient respectively. The assumed Rosseland model is quite accurate for optically-thick media for the present analysis where thermal radiation propagates a limited distance prior to encountering scattering or absorption. The refractive index of the fluidparticle suspension is assumed to be constant, intensity within the fluid is nearly isotropic and uniform, and furthermore wavelength regions exist where the optical thickness is usually more than five. Eqn. (13) results in a highly nonlinear energy equation in $T$ and it is difficult to obtain a solution. However, researchers have resolved this problem by assuming small temperature differences [46] with in the fluid flow. In this situation, Rosseland's model can be linearized about ambient temperature $T_{\infty}$ if the difference in the temperature with in the flow such that $T^{4}$ can be expressed as linear combination of the temperature. Using Taylor's series expansion about $T$ the expansion of $T^{4}$ can be written as follows, neglecting higher order terms:

$T^{4}=T_{\infty}^{4}+4 T_{\infty}^{3}\left(T-T_{\infty}\right)+6 T_{\infty}^{2}\left(T-T_{\infty}\right)^{2}+\ldots$

Neglecting higher order terms beyond the first degree in $\left(T-T_{\infty}\right)$, we have:

$T^{4} \cong 4 T_{\infty}^{3} T-3 T_{\infty}^{4}$

Differentiating Eq. (13) w.r.t $y^{\prime}$ and using (15), we obtain:

$$
\frac{\partial q_{r}}{\partial y^{\prime}}=-\frac{-16 T_{\infty}^{3} \bar{\sigma}}{3 \bar{k}} \frac{\partial^{2} T}{\partial y^{\prime}}
$$

Now simply replacing Eq. (13) with $T_{\infty}^{3}$, Eq. (9) can be expressed as follows:

$$
\frac{\partial T}{\partial t^{\prime}}+v^{\prime} \frac{\partial T}{\partial y^{\prime}}=\frac{\kappa}{\rho C_{p}}\left(1+\frac{16 \bar{\sigma}}{3 \bar{k} \kappa} T_{\infty}^{3}\right) \frac{\partial^{2} T}{\partial y^{\prime 2}}-\frac{Q^{\prime}}{\rho C_{p}}\left(T_{w}-T_{\infty}\right)+\frac{Q_{1}^{\prime}}{\rho C_{p}}\left(C-C_{\infty}\right)+\frac{\mu}{\rho C_{p}}\left(\frac{\partial u^{\prime}}{\partial y^{\prime}}\right)^{2}+\frac{\sigma B_{o}^{2}}{\rho C_{p}} u^{\prime 2}
$$

Introducing the following non-dimensional variables: 


$$
\begin{aligned}
& u=\frac{u^{\prime}}{U_{0}}, v=\frac{v^{\prime}}{V_{0}}, y=\frac{V_{0} y^{\prime}}{v}, t=\frac{t^{\prime} V_{0}^{2}}{v}, U_{p}=\frac{u_{p}}{U_{0}}, \omega=\frac{v \omega^{\prime}}{U_{0} V_{0}}, n=\frac{v n^{\prime}}{V_{0}^{2}}, \\
& \theta=\frac{\left(T-T_{\infty}\right)}{\left(T_{w}-T_{\infty}\right)}, \phi=\frac{\left(C-C_{\infty}\right)}{\left(C_{w}-C_{\infty}\right)}, j=\frac{j^{\prime} V_{0}^{2}}{v^{2}}, M=\frac{\sigma B_{o}^{2}}{\rho V_{0}^{2}}, K=\frac{V_{o}^{2} K^{\prime}}{v^{2}}, \\
& \left.\operatorname{Pr}=\frac{v \rho C p}{\kappa}=\frac{\mu C p}{\kappa}, \quad S c=\frac{v}{D}, \quad G r=\frac{g \beta_{f}\left(T_{w}-T_{\infty}\right) v}{U_{o} V_{o}^{2}}, G m=\frac{g \beta_{c}\left(C_{w}-C_{\infty}\right) v}{U_{0} V_{0}^{2}},\right\} \\
& F=\frac{16 \bar{\sigma} T_{\infty}^{3}}{3 k \bar{k}}, H=\frac{Q^{\prime} v^{2}}{V_{o}^{2} \kappa}, H_{1}=\frac{Q_{1}^{\prime}\left(C_{w}-C_{\infty}\right)}{V_{o}^{2}\left(T_{w}-T_{\infty}\right)}, E c=\frac{U_{o}^{2}}{C_{p}\left(T_{w}-T_{\infty}\right)}, \\
& K r=\frac{K_{r}^{\prime} v}{V_{o}^{2}}, \gamma=\left(\mu+\frac{\Lambda}{2}\right) j^{\prime}=\mu j^{\prime}\left(1+\frac{\beta}{2}\right), \quad \beta=\frac{\Lambda}{\mu}
\end{aligned}
$$

Where all quantities with a prime are dimensionless, In view of equations (11)- (18) the governing equations (7) -(10) after dropping primes are transformed into the following system of unsteady coupled, dimensionless partial differential equations:

$$
\begin{aligned}
& \frac{\partial u}{\partial t}-\left(1+\varepsilon A e^{n t}\right) \frac{\partial u}{\partial y}=(1+\beta) \frac{\partial^{2} u}{\partial y^{2}}+G r \theta \cos \alpha+G \operatorname{cocos} \alpha-\left(M+\frac{(1+\beta)}{K}\right) u+2 \beta \frac{\partial \omega}{\partial y} \\
& \frac{\partial \omega}{\partial t}-\left(1+\varepsilon A e^{n t}\right) \frac{\partial \omega}{\partial y}=\frac{1}{\eta} \frac{\partial^{2} \omega}{\partial y^{2}} \\
& \frac{\partial \theta}{\partial t}-\left(1+\varepsilon A e^{n t}\right) \frac{\partial \theta}{\partial y}=\frac{1}{P r}(1+F) \frac{\partial^{2} \theta}{\partial y^{2}}-\frac{H}{P r} \theta+H_{1} \phi+E c\left(\left(\frac{\partial u}{\partial y}\right)^{2}+M u^{2}\right) \\
& \frac{\partial \phi}{\partial t}-\left(1+\varepsilon A e^{n t}\right) \frac{\partial \phi}{\partial y}=\frac{1}{S c} \frac{\partial^{2} \phi}{\partial y^{2}}+K r \phi
\end{aligned}
$$

The transformed initial and boundary conditions can be written in non-dimensional form as follows:

$$
\begin{array}{ll}
t \leq 0: & \begin{cases}u=0, \quad \omega=0, T=T_{\infty}, \quad C=C_{\infty} & \text { for all } y \geq 0\end{cases} \\
t>0: & \begin{cases}u=U_{p}, \omega=-n_{1} \frac{\partial u}{\partial y}, \quad \theta=\left(1+\varepsilon e^{n t}\right), \phi=\left(1+\varepsilon e^{n t}\right) & \text { at } y=0 \\
u \rightarrow 0, \omega \rightarrow 0, \quad \theta \rightarrow 0, \quad \phi \rightarrow 0 & \text { as } y \rightarrow \infty\end{cases}
\end{array}
$$

where $\eta=\mu j^{\prime} / \gamma=2 /(2+\beta)$ is the dimensionless gyro-viscosity micropolar material parameter, $U_{p}$ is the velocity of the moving plate, $\theta$ is dimensionless temperature function, $\phi$ is dimensionless species concentration, 
and $\beta, M, K, F, H, H_{1}, \gamma$ are the Eringen micropolar vortex viscosity parameter, Magnetic parameter, permeability of the porous medium, thermal radiation-conduction parameter, heat absorption parameter, radiation absorption parameter and chemical reaction parameter respectively. $P r, S c, G r, G m, E c$ are the Prandtl number, Schmidt number, thermal Grashof number, species Grashof number and Eckert number respectively. The mathematical statement of the problem is now complete and embodies the solution of equations (19) - (22) under the prescribed boundary conditions (23).

\section{NUMERICAL SOLUTION}

In the present study, the fourth order Runge-Kutta with shooting technique has been employed to solve the transformed unsteady transport problem as described by the non-dimensional governing equations (19) subjected to boundary conditions (23) for different values of controlling parameters. To solve these equations (19)- (22) we initially apply the following perturbation equations for velocity, angular velocity (micro-rotation component), temperature and concentration with perturbation parameter $\varepsilon$ assuming $\varepsilon<<1$.

$$
\begin{aligned}
& u(y, t)=u_{0}(y)+\varepsilon e^{n t} u_{1}(y)+o\left(\varepsilon^{2}\right) \\
& \omega(y, t)=\omega_{0}(y)+\varepsilon e^{n t} \omega_{1}(y)+o\left(\varepsilon^{2}\right) \\
& \theta(y, t)=\theta_{0}(y)+\varepsilon e^{n t} \theta_{1}(y)+o\left(\varepsilon^{2}\right) \\
& \left.\phi(y, t)=\phi_{0}(y)+\varepsilon e^{n t} \phi_{1}(y)+o\left(\varepsilon^{2}\right)\right)
\end{aligned}
$$

Substituting equation (24) in equations (19) -(22) and in the boundary condition (23) and then equating the coefficients of $\varepsilon^{0}$ and $\varepsilon^{1}$, yields:

\section{Zeroth order}

$$
\begin{aligned}
& (1+\beta) \frac{\partial^{2} u_{0}}{\partial y^{2}}+\frac{\partial u_{0}}{\partial y}+G r \theta_{0} \cos \alpha+G c \phi_{0} \cos \alpha-\left(M+\frac{(1+\beta)}{K}\right) u_{0}+2 \beta \frac{\partial \omega_{0}}{\partial y}=0 \\
& \frac{\partial^{2} \omega_{0}}{\partial y^{2}}+\eta \frac{\partial \omega_{0}}{\partial y}=0 \\
& \frac{1}{P r}(1+F) \frac{\partial^{2} \theta_{0}}{\partial y^{2}}+\frac{\partial \theta_{0}}{\partial y}-\frac{H}{P r} \theta_{0}+H_{1} \phi_{0}+E c\left(\left(\frac{\partial u_{0}}{\partial y}\right)^{2}+M u_{0}^{2}\right)=0 \\
& \frac{1}{S c} \frac{\partial^{2} \phi_{0}}{\partial y^{2}}+\frac{\partial \phi_{0}}{\partial y}+K r \phi_{0}=0
\end{aligned}
$$




\section{First order}

$(1+\beta) \frac{\partial^{2} u_{1}}{\partial y^{2}}+\frac{\partial u_{1}}{\partial y}+G r \theta_{1} \cos \alpha+G c \phi_{1} \cos \alpha-\left(M+\frac{(1+\beta)}{K}\right) u_{0}+A \frac{\partial u_{0}}{\partial y}+2 \beta \frac{\partial \omega_{1}}{\partial y}=0$

$\frac{\partial^{2} \omega_{1}}{\partial y^{2}}+\eta \frac{\partial \omega_{1}}{\partial y}-n \eta \omega_{1}+A \eta \frac{\partial \omega_{0}}{\partial y}=0$

$\frac{1}{P r}(1+F) \frac{\partial^{2} \theta_{1}}{\partial y^{2}}+\frac{\partial \theta_{1}}{\partial y}-\frac{H}{P r} \theta_{1}-n \theta_{1}+H_{1} \phi_{1}+A \frac{\partial \theta_{0}}{\partial y}+E c\left(2 \frac{\partial u_{0}}{\partial y} \frac{\partial u_{1}}{\partial y}+2 M u_{0} u_{1}\right)=0$

$\frac{1}{S c} \frac{\partial^{2} \phi_{1}}{\partial y^{2}}+\frac{\partial \phi_{1}}{\partial y}+(K r-n) \phi_{1}+A \eta \frac{\partial \phi_{0}}{\partial y}=0$

$y=0: \quad\left\{u_{0}=U_{P}, u_{1}=0, \omega_{0}=-n_{1} \frac{\partial u_{0}}{\partial y}, \omega_{1}=-n_{1} \frac{\partial u_{1}}{\partial y}, \theta_{0}=1, \theta_{1}=1, \phi_{0}=1, \phi_{1}=1\right.$

$y \rightarrow \infty:\left\{\begin{array}{l}\text { and } \\ u_{0} \rightarrow 0, u_{1} \rightarrow 0, \omega_{0} \rightarrow 0, \omega_{1} \rightarrow 0, \theta_{0} \rightarrow 0, \theta_{1} \rightarrow 0, \phi_{0} \rightarrow 0, \phi_{1} \rightarrow 0\end{array}\right.$

The above non-linear coupled partial differential equations (25)- (32) with the boundary condition (33) are then solved by using Runge-Kutta fourth order numerical method with shooting quadrature. There are several important wall (plate) parameters for materials processing boundary layer flows.

The skin-friction at the plate, in non-dimensional form is given by:

$C_{f}=\left.\frac{\partial u}{\partial y}\right|_{y=0}$

The couple stress coefficient at the plate, in non-dimensional form is given by:

$C_{w}=\left.\frac{\partial \omega}{\partial y}\right|_{y=0}$

Nusselt number is computed in non-dimensional form as:

$N u \operatorname{Re}_{x}{ }^{-1}=-\left.\frac{\partial \theta}{\partial y}\right|_{y=0}$

Sherwood number is evaluated in the non-dimensional form as follows:

$\operatorname{ShRe}{ }_{x}^{-1}=-\left.\frac{\partial \phi}{\partial y}\right|_{y=0}$

where $R_{x}=V_{o} x / v$ is the local suction velocity-based Reynolds number 


\section{NUMERICAL RESULTS, VALIDATION AND DISCUSSION}

The prime aim of this paper is to investigate selected parameter effects on chemically reacting micropolar unsteady dissipative magneto hydromagnetic mixed convection heat and mass transfer from an oscillatory inclined porous plate considering heat absorption, radiation absorption and Joule heating. The dictating thermal, diffusive and hydrodynamic parameters are gyro-viscosity micropolar material parameter, magnetic field parameter, permeability of the porous medium, Prandtl number, Schmidt number, thermal Grashof number, species Grashof number, thermal radiation-conduction parameter, heat absorption parameter, radiation absorption parameter, Eckert number, chemical reaction parameter and Eringen coupling number (vortex viscosity ratio parameter). Numerical computations for velocity, angular velocity, temperature and concentration are obtained by using Runge-Kutta fourth order with shooting technique. This method is well-established in the literature and therefore details are not repeated here. Readers are referred to Uddin et al. [47], Bég et al. [48] and Reddy and Sandeep [49] for details. The results of this study are compared with analytical results of Pal and Biswas [26]. Table 1, displays the comparison of computed numerical results employed by Runge-Kutta fourth order associated with shooting technique with those of analytical results obtained via a perturbation technique by Pal and Biswas [26], for local skin friction coefficient, wall couple stress coefficient, Nusselt number and Sherwood number for different values of $S c, P r, K, M, F$ and $E c$. These solutions negate angle of inclination, heat absorption and radiation absorption, since these terms were ignored in the model of Pal and Biswas [26]. Generally, very good correlation is achieved. Table $\mathbf{1}$ further shows that, as permeability, radiation and Eckert number increases, skin friction and couple stress coefficients both increase whereas the converse trend is observed in the case of Nusselt number i.e. wall heat transfer rate is reduced with increasing permeability, radiation and Eckert number. It is also noticed that as Schmidt number, Prandtl number and magnetic parameter increases there is suppression in skin friction and couple stress coefficient whereas there is an increase in Nusselt number. Furthermore, as Schmidt number decreases Sherwood number decreases. 
Table 1: Comparison of the present numerical results of $C_{f}, C_{w}, N u / R e_{x}$ and $S h / R e_{x}$ with Pal and Biswas [20] for $S c, P r, K, M, F$ and $E c$ when $\alpha=0, H=0, H_{1}=0, \beta=1, G r=5, G m=10, S c=2, K r=0.1$, $U_{p}=0.5$.

\begin{tabular}{|c|c|c|c|c|c|c|c|c|c|c|c|c|c|}
\hline & & & & & & Pal and $B$ & iswas Per & $\begin{array}{l}\text { turbation } \\
\text { 0] }\end{array}$ & olutions & Pre & sent $\mathrm{Nu}$ & cal Solu & \\
\hline Sc & $\operatorname{Pr}$ & $K$ & $M$ & $F$ & $E c$ & $C_{f}$ & $C_{w}$ & $N u / \operatorname{Re}_{x}$ & $S h / R e_{x}$ & $C_{f}$ & $C_{w}$ & $N u / R e_{x}$ & $S h / R e_{x}$ \\
\hline 2 & 1 & 5 & 2 & 0.1 & 0.001 & 0.2707133 & 0.0301309 & 0.8914258 & 1.9158002 & 0.27071296 & 0.03013088 & 0.89142571 & 1.91580023 \\
\hline 1 & 1 & 5 & 2 & 0.1 & 0.001 & 0.6682574 & 0.0745723 & 0.8914186 & 0.8975272 & 0.66825737 & 0.07457226 & 0.89141873 & 0.89752718 \\
\hline 0.5 & 1 & 5 & 2 & 0.1 & 0.001 & 1.1339602 & 0.1264654 & 0.8914083 & 0.3664133 & 1.13396016 & 0.12646545 & 0.89140840 & 0.36641332 \\
\hline 2 & 3 & 5 & 2 & 0.1 & 0.001 & -0.8333037 & -0.0927355 & 2.6738615 & 1.9158002 & -0.8333030 & 0.09273561 & 2.67386143 & \\
\hline 2 & 5 & 5 & 2 & 0.1 & 0.001 & -1.2005236 & -0.1335068 & 4.4563727 & 1.9158002 & -1.20052371 & -0.13350701 & 4.45637266 & 1.91580020 \\
\hline 2 & 1 & 3 & 2 & 0.1 & 0.001 & -0.0745329 & 0.0083268 & 0.8914279 & 1.9158002 & 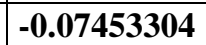 & 0.00832675 & 0.89142811 & 1.91580020 \\
\hline 2 & 1 & 1 & 2 & 0.1 & 0.001 & -0.698520 & -0.0776972 & 0.8914325 & 1.9158002 & -0.69851972 & -0.07769716 & 0.89143240 & 1.91580020 \\
\hline 2 & 1 & 5 & 3 & 0.1 & 0.001 & -0.3851199 & -0.0427542 & 0.8914313 & 1.9158002 & \begin{tabular}{|c|}
-0.38512010 \\
\end{tabular} & -0.04275425 & 0.89143120 & 1.91580020 \\
\hline 2 & 1 & 5 & 4 & 0.7 & 0.001 & -0.8853874 & -0.0985380 & 0.8914328 & 1.9158002 & \begin{tabular}{|l|l|}
-0.85538750 \\
\end{tabular} & 0.09853814 & 0.89143276 & 1.91580020 \\
\hline 2 & 1 & 5 & 2 & 0.3 & 0.001 & 0.5054170 & 0.0560755 & 0.7216151 & 1.9158002 & $0.50 S 541692$ & 0.05607532 & 0.72161508 & 1.91580020 \\
\hline 2 & 1 & 5 & 2 & 0.5 & 0.001 & 0.6920367 & 0.0770952 & 0.6061646 & 1.9158002 & 0.69203672 & 0.07709517 & $\mid 0.60616456$ & 1.91580020 \\
\hline 2 & 1 & 5 & 2 & 0.1 & 0.005 & 0.2717460 & 0.0301607 & 0.8912639 & 1.9158002 & 0.27174605 & 0.03016064 & 0.89126412 & 1.91580020 \\
\hline 2 & 1 & 5 & 2 & 0.1 & 0.007 & 0.2723846 & 0.0301891 & 0.8911055 & 1.9158002 & $\mathbf{0 . 2 7 2 3 8 4 5 7}$ & 0.03018908 & 0.89110549 & 1.91580020 \\
\hline 2 & 1 & 5 & 2 & 0.1 & 0.1 & 0.2734951 & 0.0302488 & 0.8907710 & 1.9158002 & 0.27349505 & 0.03024892 & 0.89077151 & 1.91580020 \\
\hline
\end{tabular}

The influence of $\alpha, M, G r, G c, \beta, U p, K, F, H, H_{1}, E c, S c$, and $K r$ on the micropolar fluid velocity, microrotation, temperature and concentration distributions are presented graphically in Figures 2-18. Computations are performed with the following default values of all parameters: $\varepsilon=0.01, t=1, \eta=0.22, A=1$, $k=1, n=0.1$, and $\operatorname{Pr}=1$ while $\alpha, M, G r, G c, \beta, U p, K, F, H, H_{1}, E c, S c, K r$ are varied over a range, which are listed in the figure legends. 


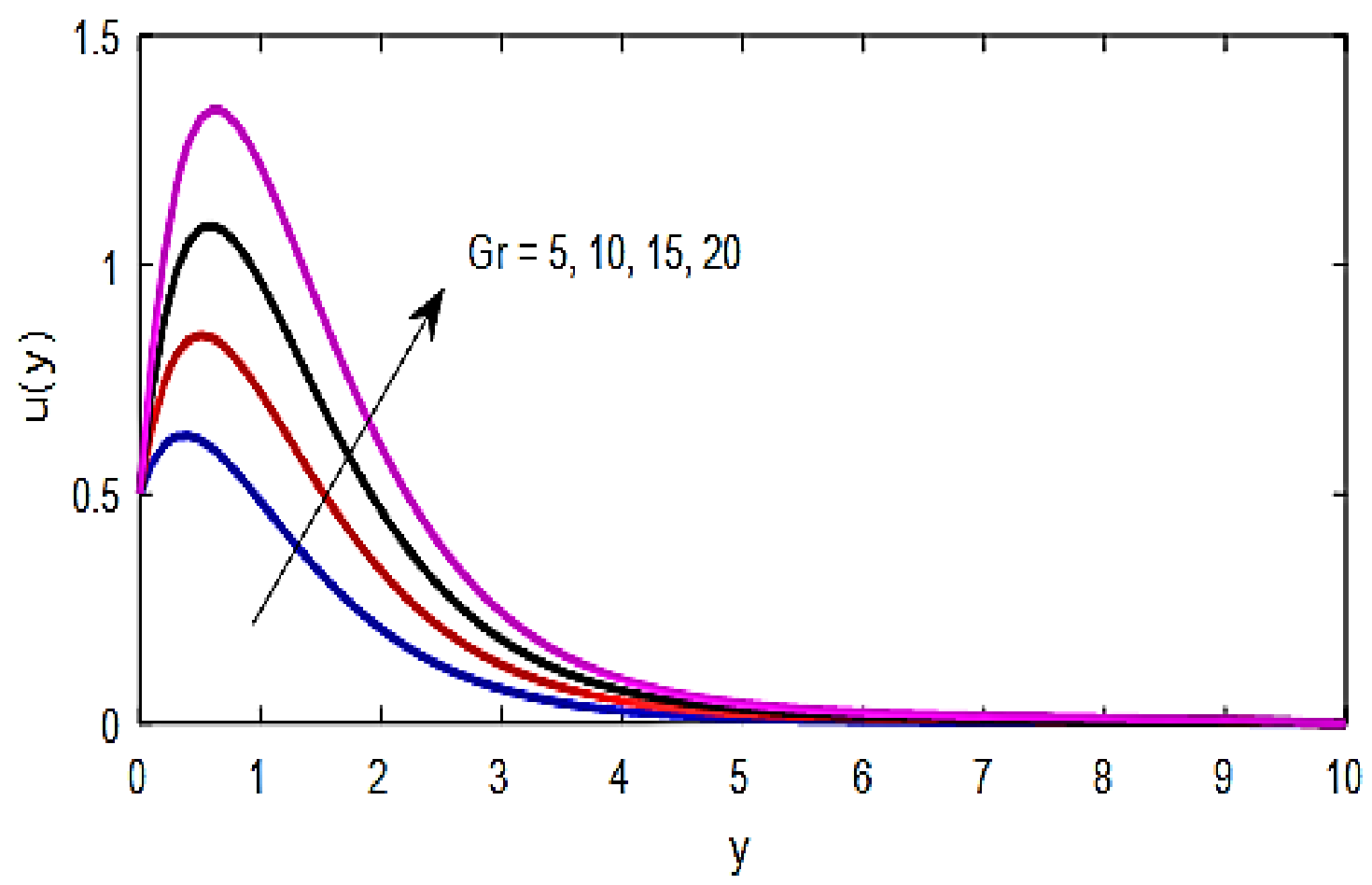

Figure 2: Velocity distribution for various Grashof numbers $G r$ when $K=5, M=2, G c=5$, $\alpha=\pi / 4, \beta=1, H=1, H_{1}=1, F=0.1, E c=0.01, S c=2, K r=0.1, U_{p}=0.5$.

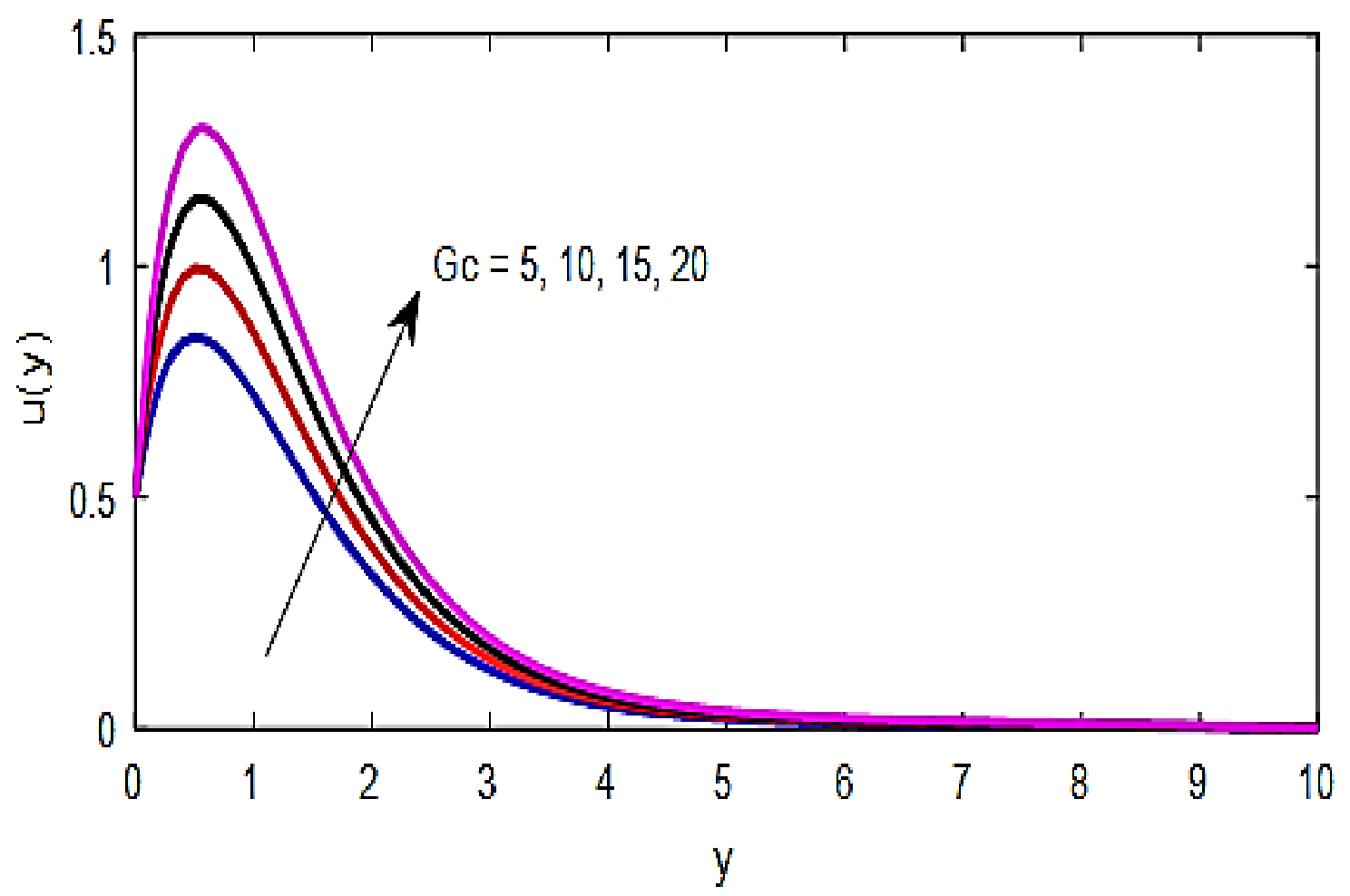

Figure 3:Velocity distribution for various species Grashof numbers $G c$ when $K=5, M=2$, $G r=10, \alpha=\pi / 4, \beta=1, H=1, H_{1}=1, F=0.1, E c=0.01, S c=2, K r=0.1, U_{p}=0.5$. 


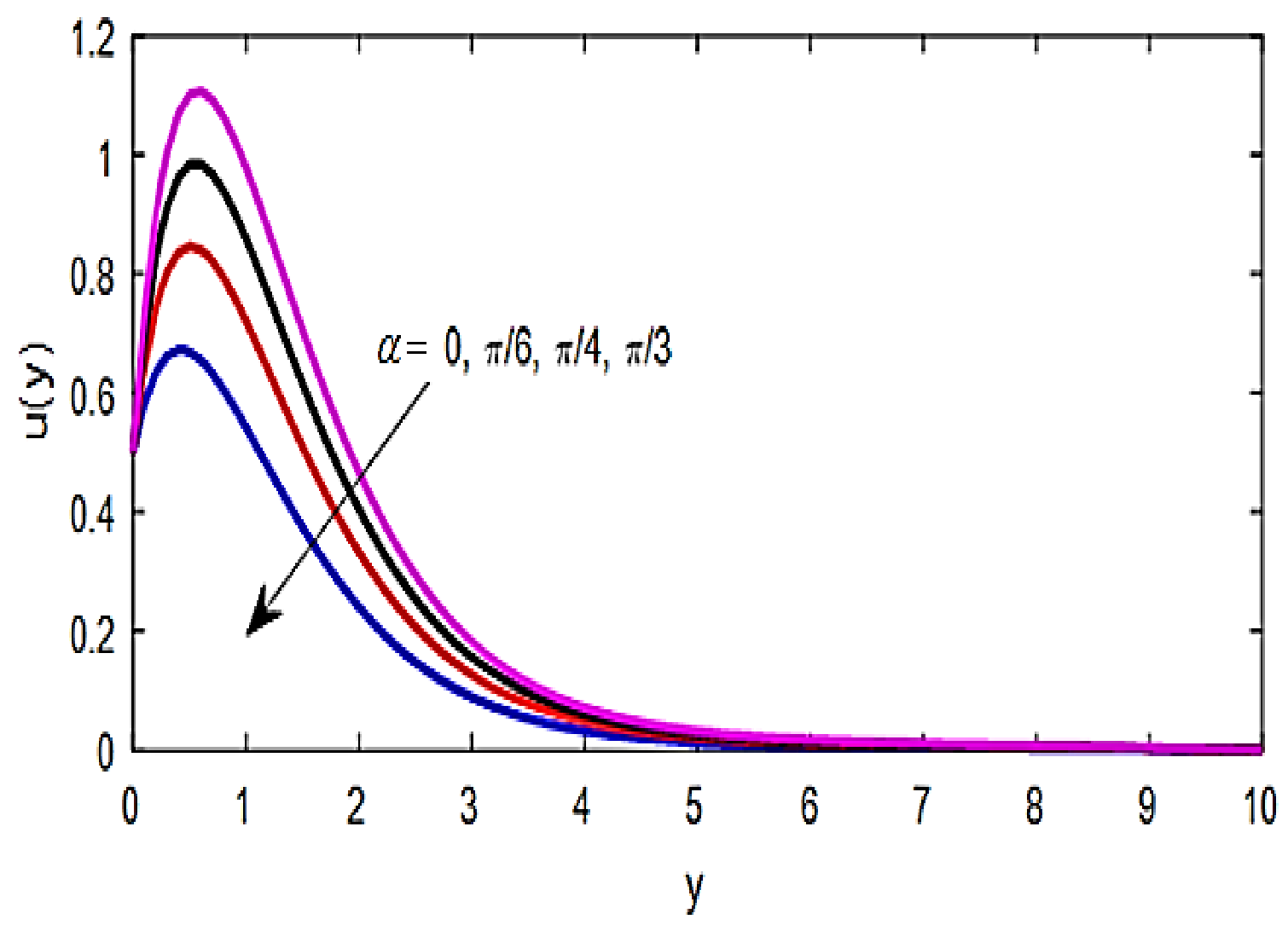

Figure 4: Velocity distribution for angle of inclination $\alpha$ when $K=5, M=2, G r=10, G c=5$, $\beta=1, H=1, H_{1}=1, F=0.1, E c=0.01, S c=2, K r=0.1, U_{p}=0.5$.

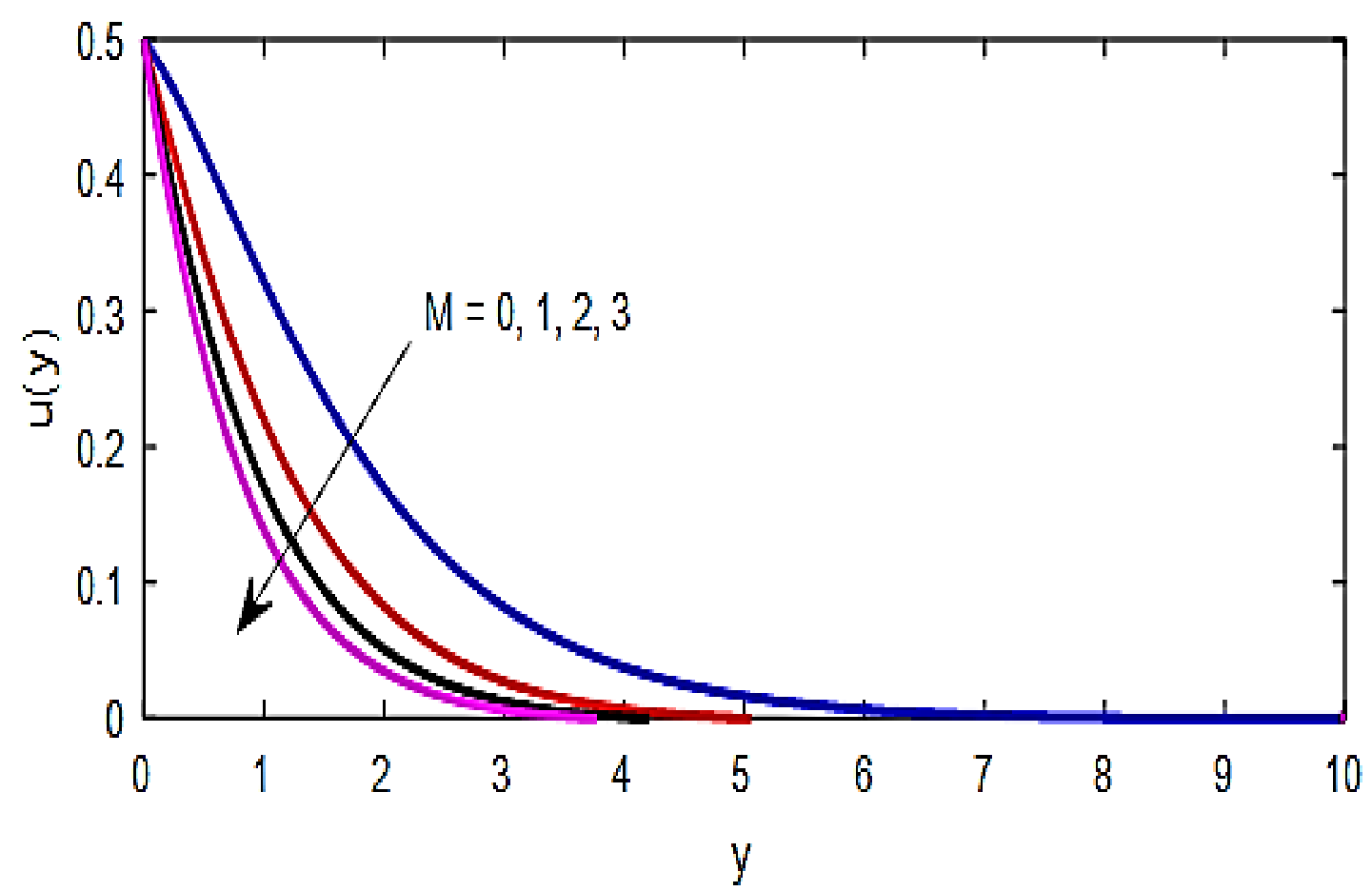

Figure 5: Velocity distribution for Magnetic field parameter $M$ when $K=0.5, G r=10$, $G c=5, \alpha=\pi / 4, \beta=1, H=1, H_{1}=1, F=0.1, E c=0.01, S c=2, K r=0.1, U_{p}=0.5$. 


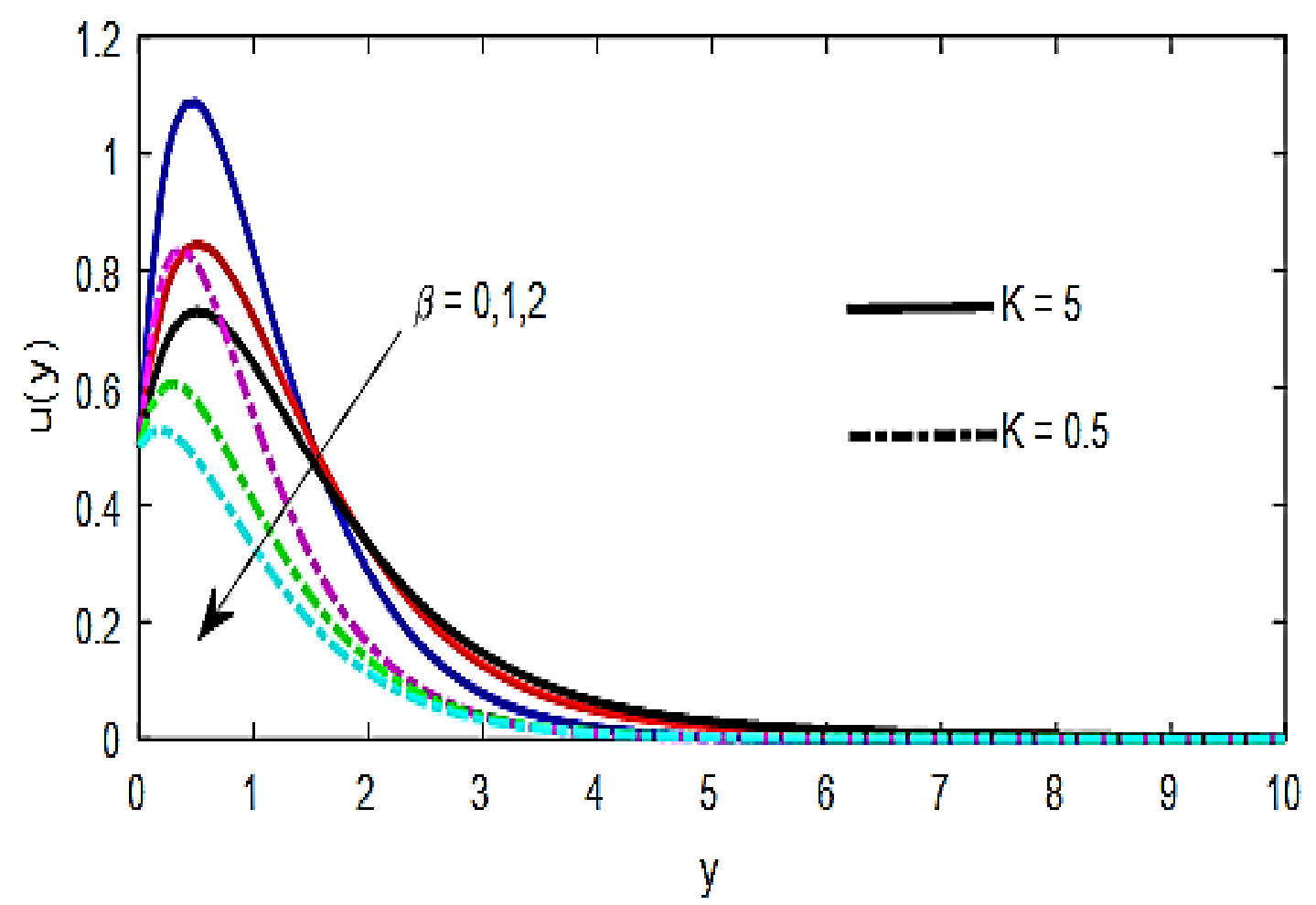

Figure 6: Velocity distribution for Eringen micropolar vortex viscosity parameter $\beta$ when $M=2, G r=10, G c=5, \alpha=\pi / 4, H=1, H_{1}=1, F=0.1, E c=0.01, S c=2, K r=0.1, U_{p}=0.5$.

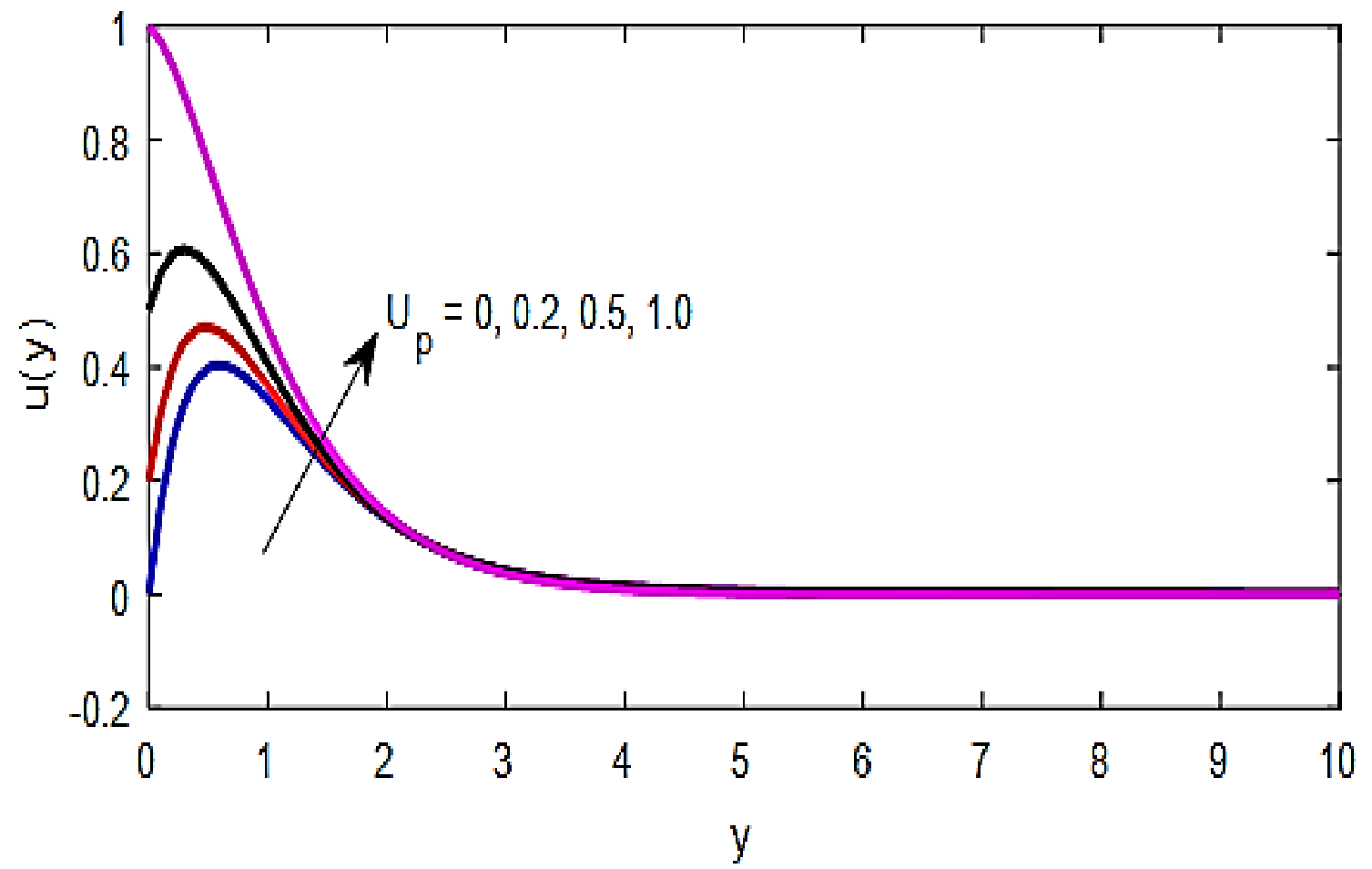

Figure 7: Velocity distribution for plate moving velocity parameter $U_{P}$ when $K=0.5, M=2$, $G r=10, G c=5, \alpha=\pi / 4, \beta=1, H=1, H_{1}=1, F=0.1, E c=0.01, S c=2, K r=0.1$. 


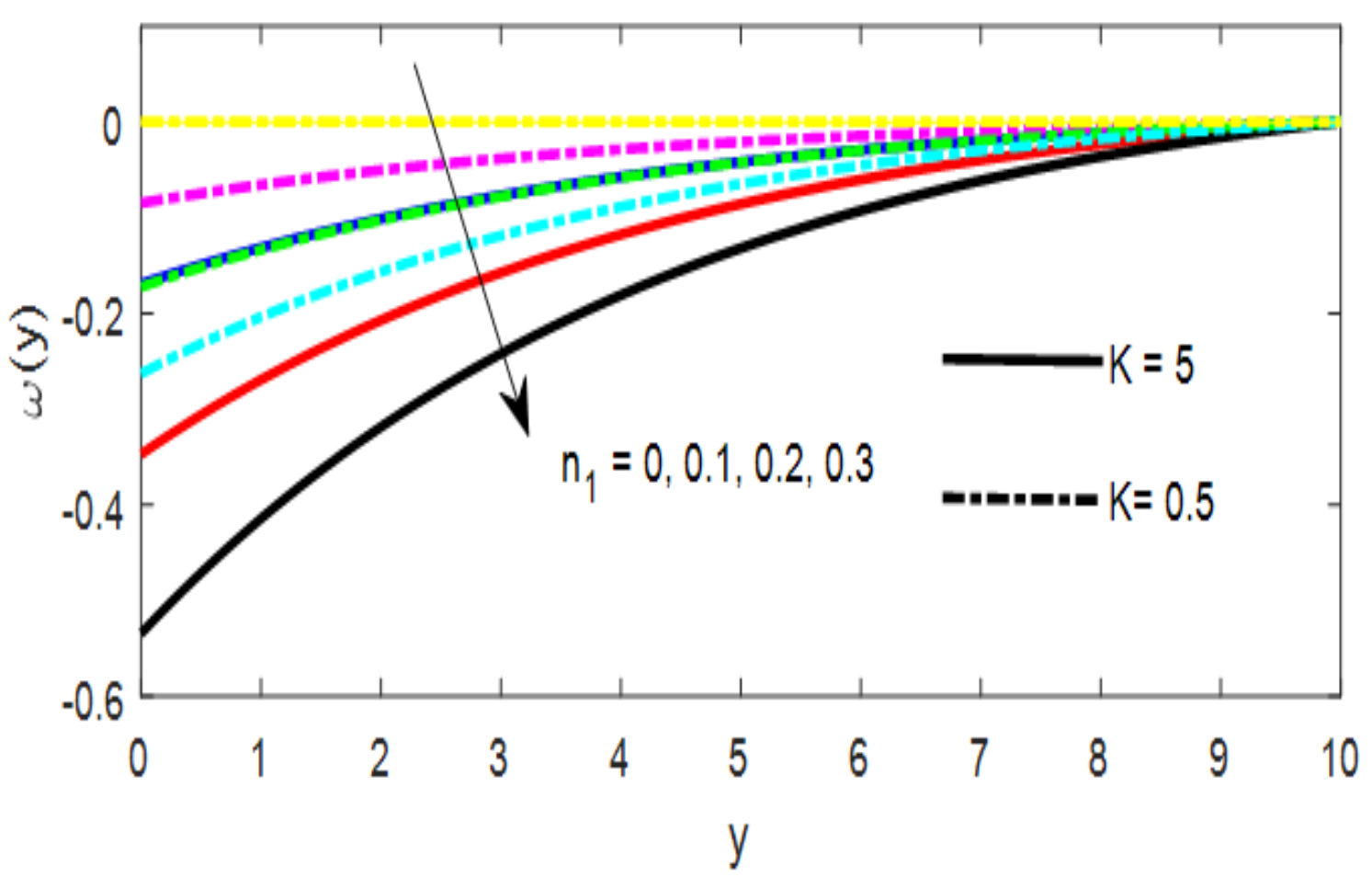

Figure 8: Angular velocity distribution for micro-gyration parameter $n_{1}$ when $M=2, G r=10$,

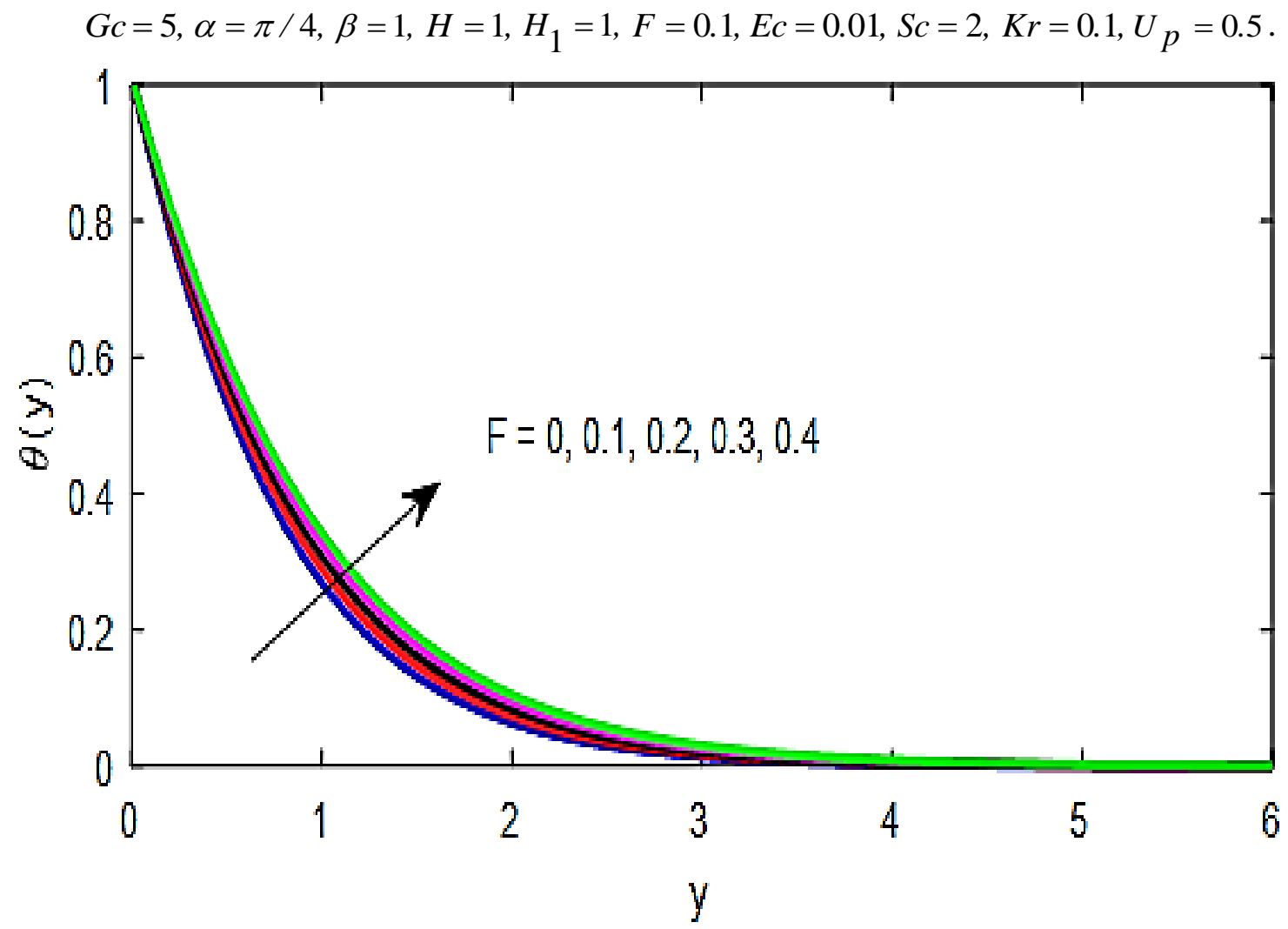

Figure 9: Temperature distribution for radiation parameter $F$ when $K=0.5, M=2, G r=10$, $G c=5, \alpha=\pi / 4, \beta=1, H=1, H_{1}=1, E c=0.01, S c=2, K r=0.1, U_{p}=0.5$. 


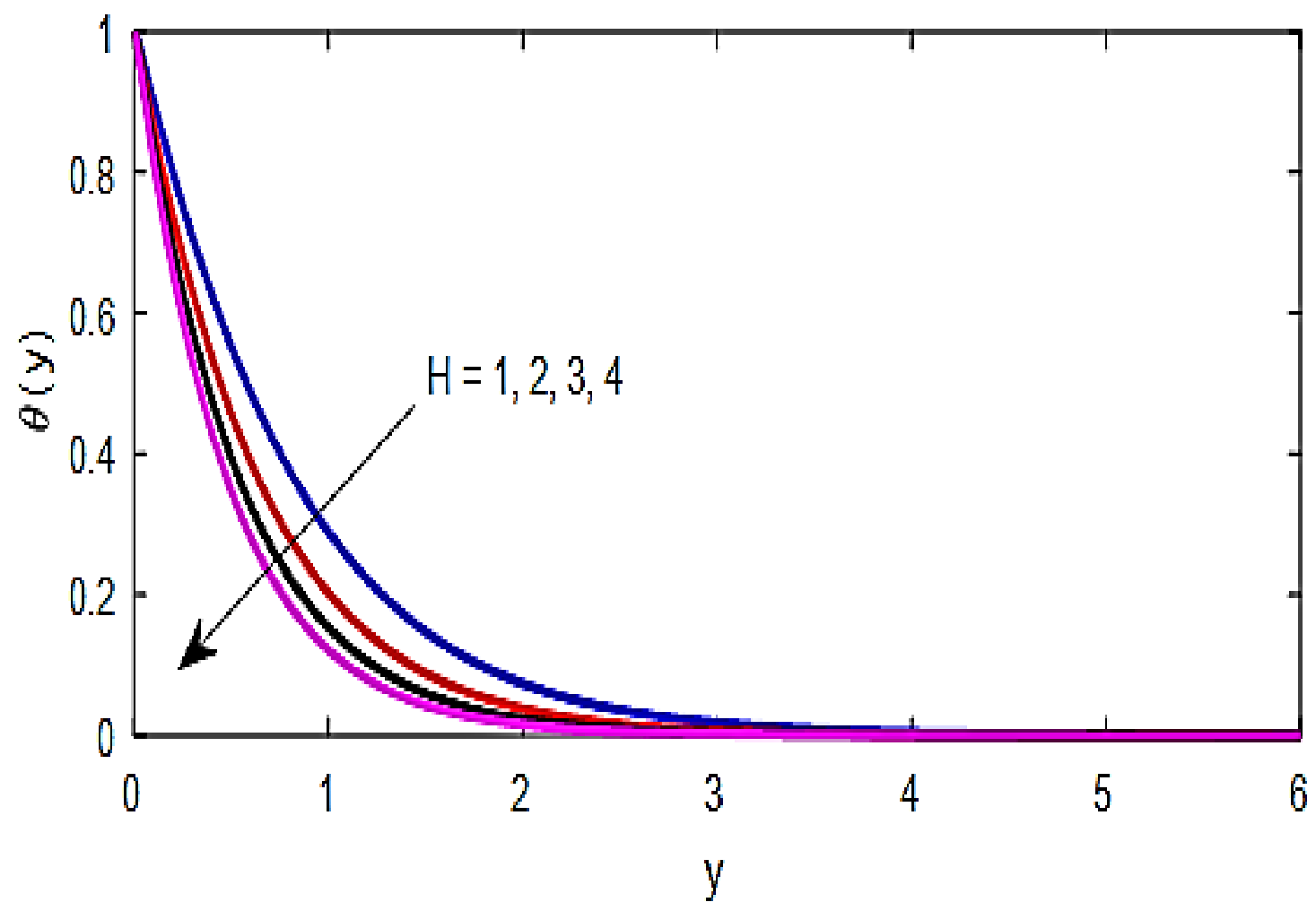

Figure 10: Temperature distribution for heat source parameter $H$ when $K=0.5, M=2$, $G r=10, G c=5, \alpha=\pi / 4, \beta=1, F=0.1, H_{1}=1, E c=0.01, S c=2, K r=0.1, U_{p}=0.5$.

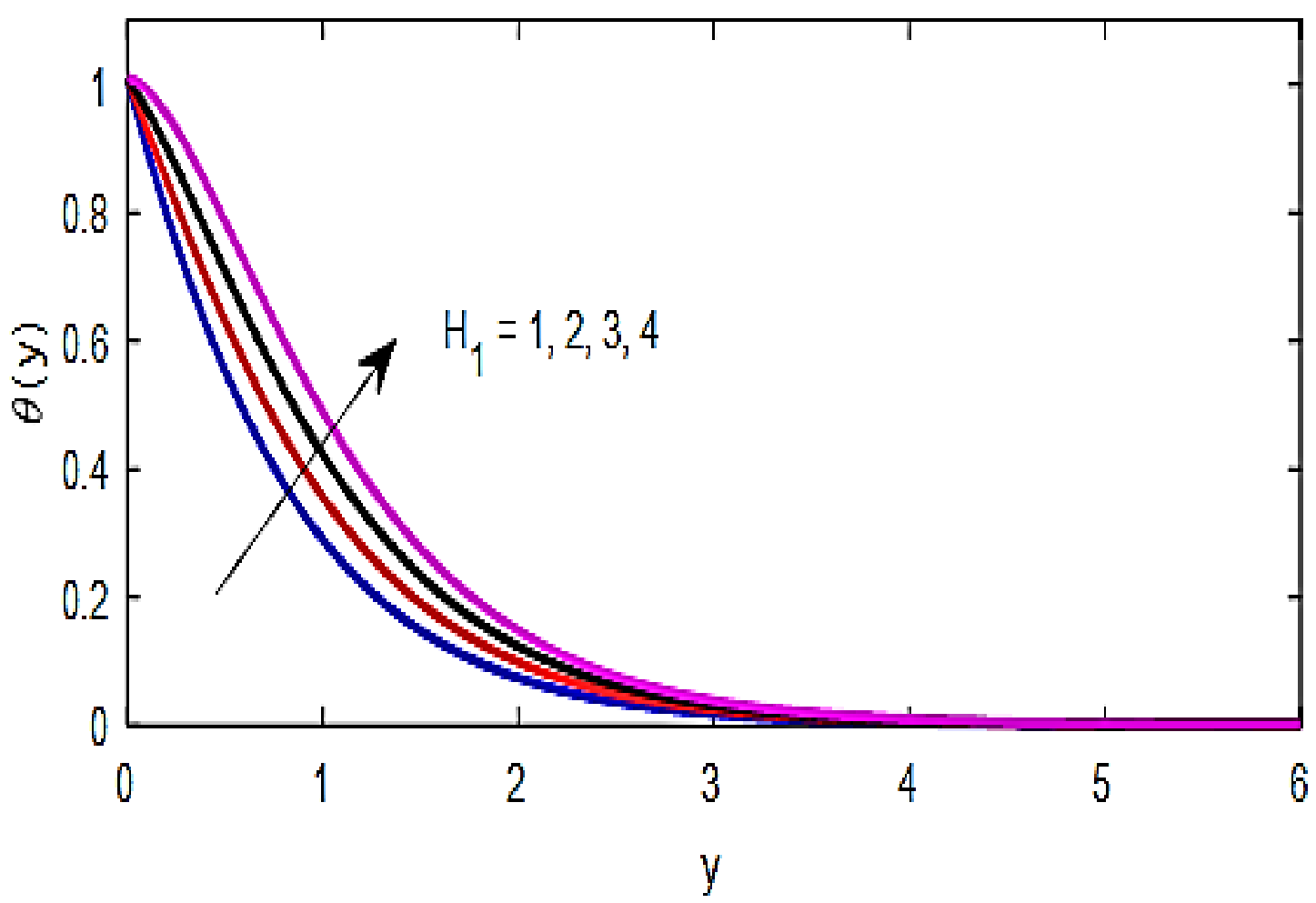

Figure 11: Temperature distribution for radiation absorption parameter $H_{1}$ when $K=0.5$, $M=2, G r=10, G c=5, \alpha=\pi / 4, \beta=1, F=0.1, H=1, E c=0.01, S c=2, K r=0.1, U_{p}=0.5$. 


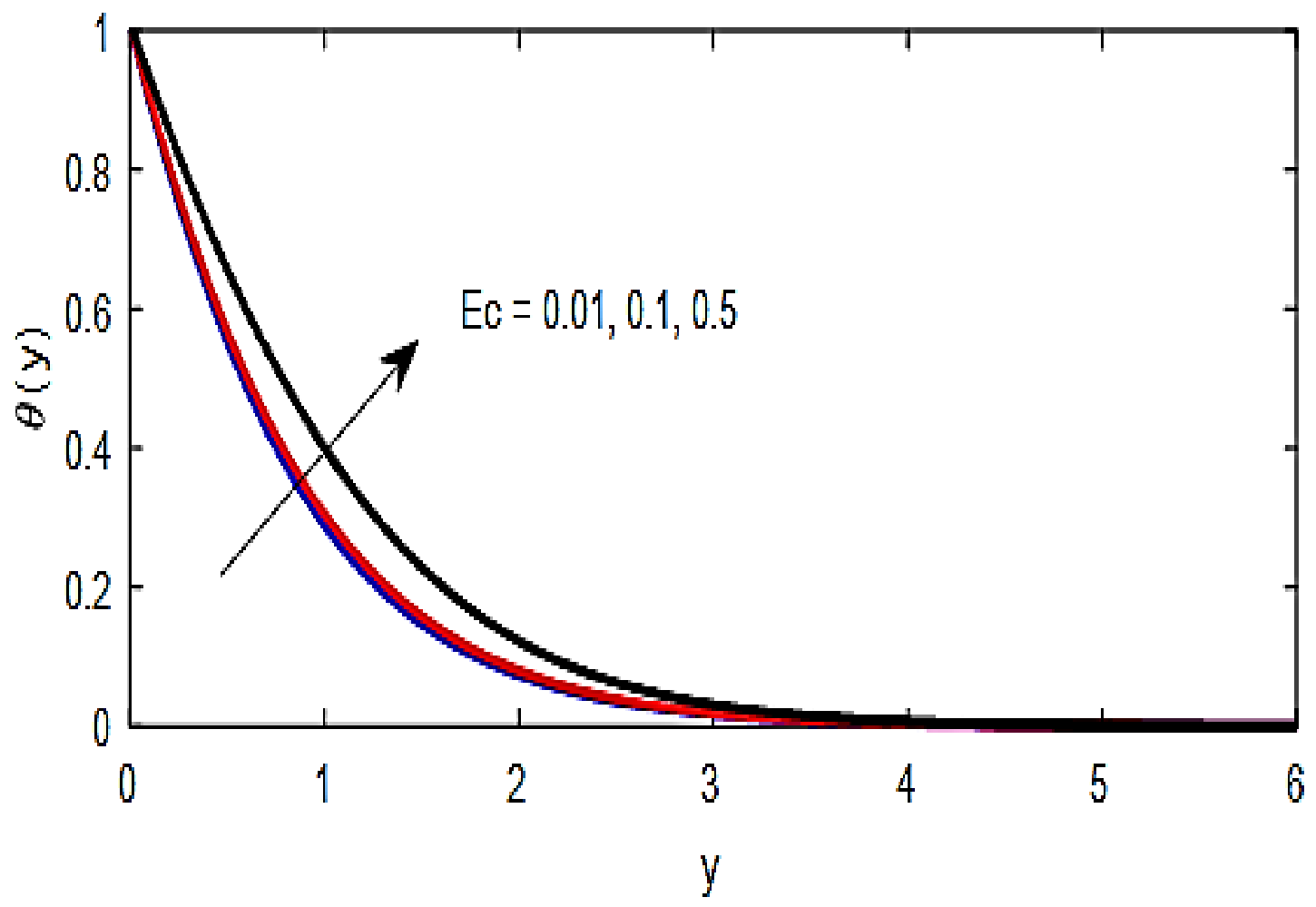

Figure 12: Temperature distribution for Eckert number $E c$ when $K=0.5, M=2, G r=10$, $G c=5, \alpha=\pi / 4, \beta=1, F=0.1, H=1, H_{1}=1, S c=2, K r=0.1, U_{p}=0.5$.

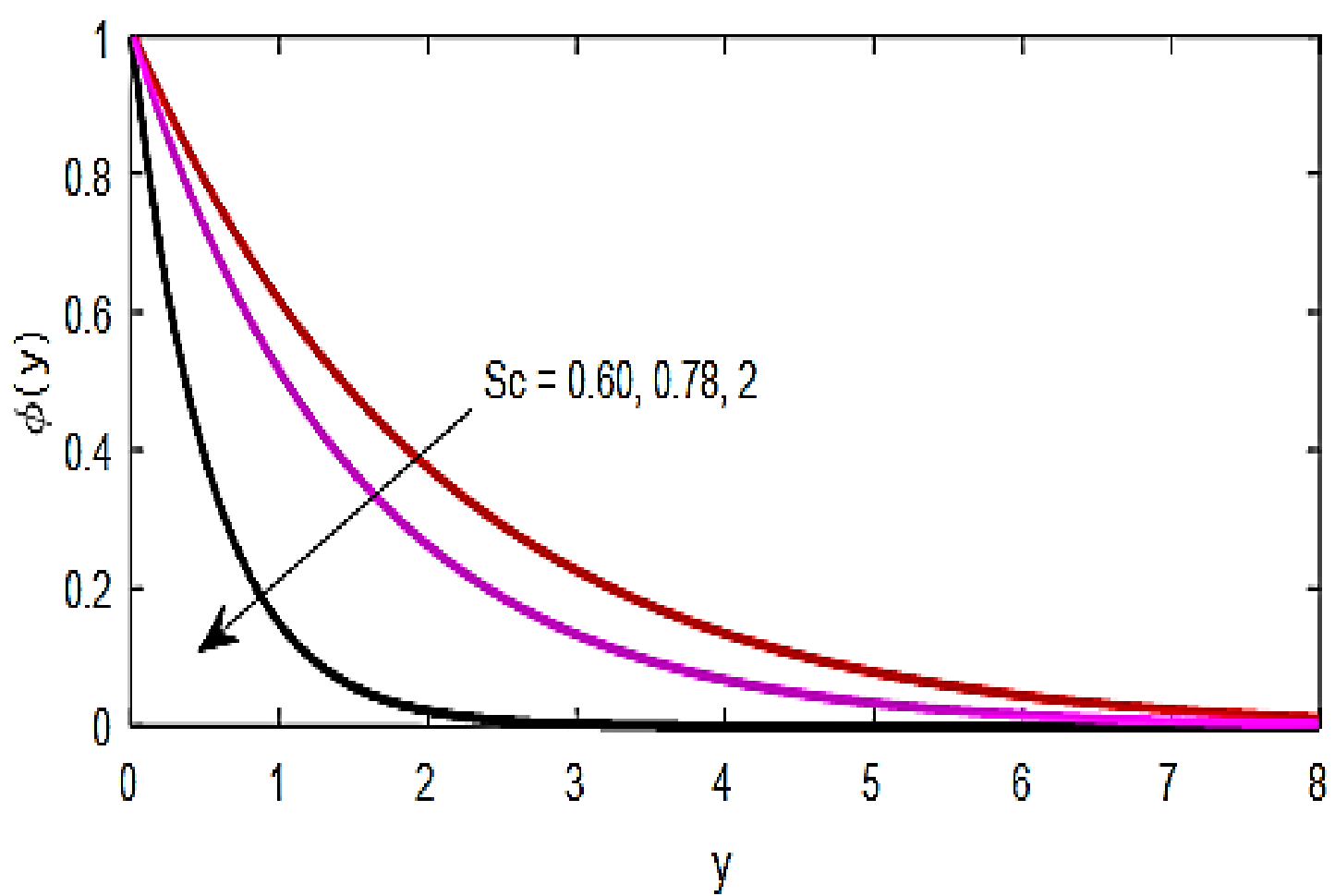

Figure 13: Concentration distribution for Schmidt number $S c$ when $K=0.5, M=2, G r=10$, $G c=5, \alpha=\pi / 4, \beta=1, F=0.1, H=1, H_{1}=1, K r=0.1, U_{p}=0.5$. 


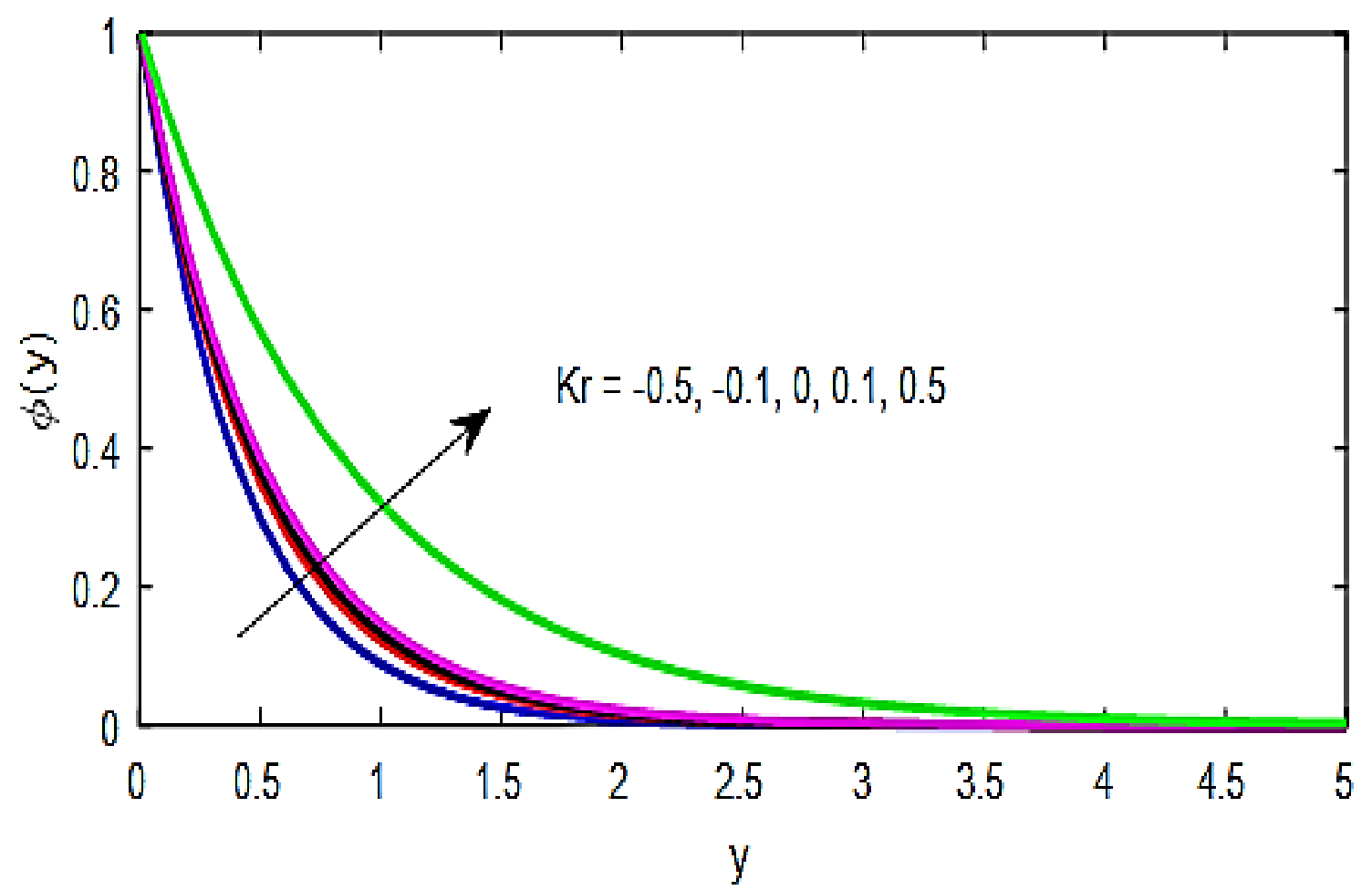

Figure 14: Concentration distribution for first order chemical reaction $K r$ when $K=0.5$, $M=2, G r=10, G c=5, \alpha=\pi / 4, \beta=1, F=0.1, H=1, H_{1}=1, S c=2, U_{p}=0.5$.

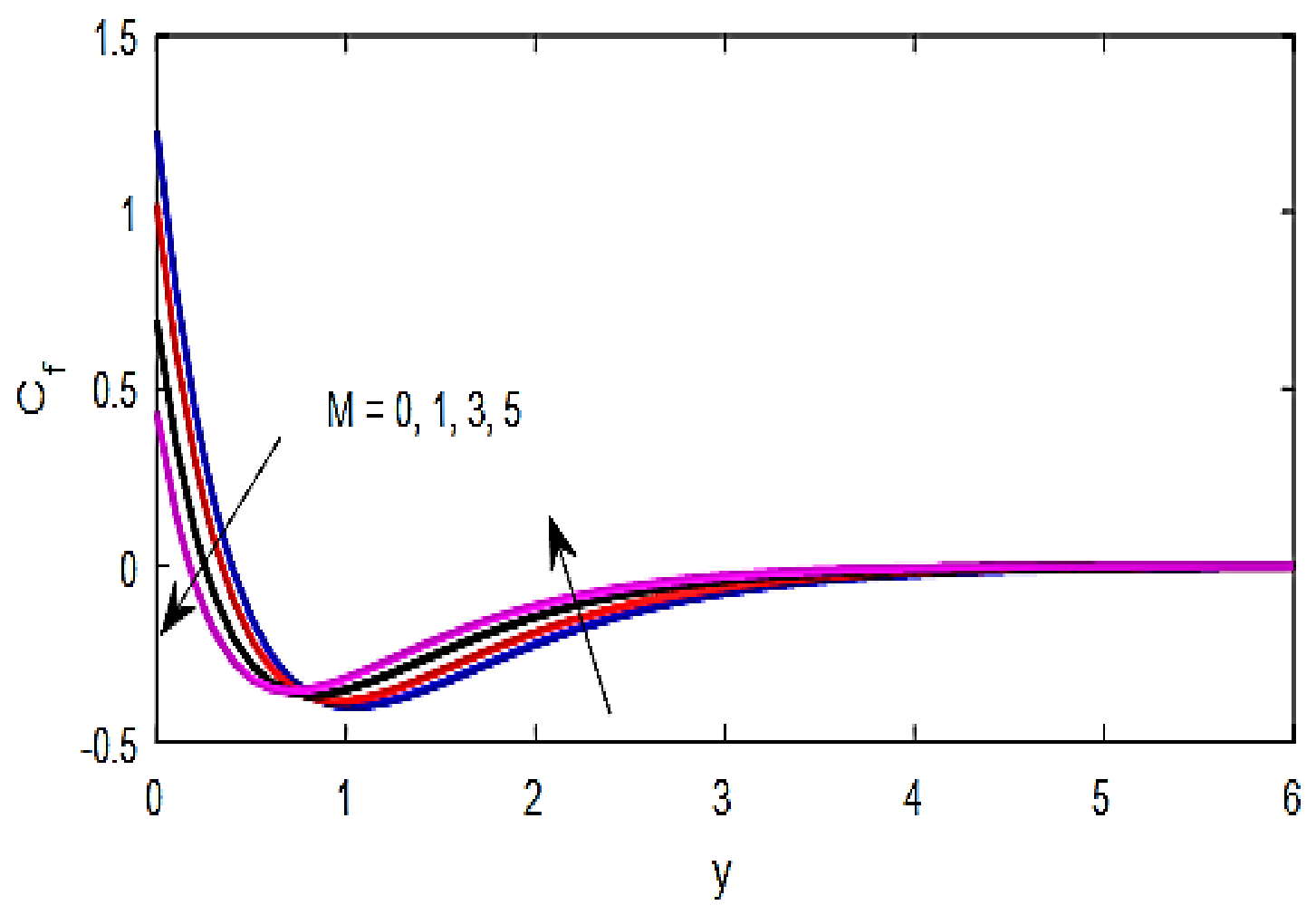

Figure 15: Skin friction coefficient $C_{f}$ for different values of $M$ when $K=0.5, G r=10$, $G c=5, \alpha=\pi / 4, \beta=1, F=0.1, H=1, H_{1}=1, S c=2, K r=0.1, U_{p}=0.5$. 


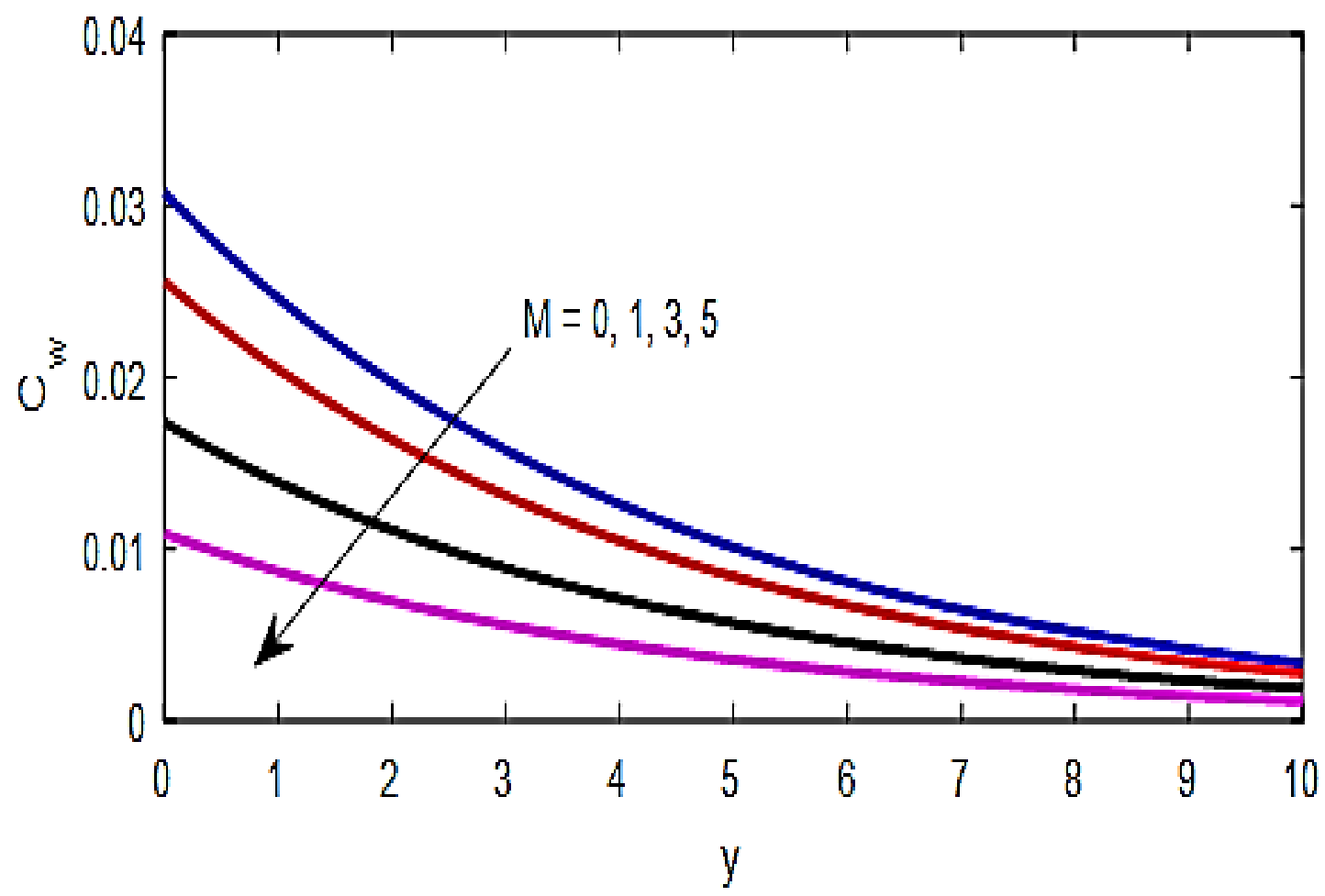

Figure 16: Wall couple stress coefficient $C_{w}$ for different values of $M$ when $K=0.5$, $G r=10, G c=5, \alpha=\pi / 4, \beta=1, F=0.1, H=1, H_{1}=1, S c=2, K r=0.1, U_{p}=0.5$.

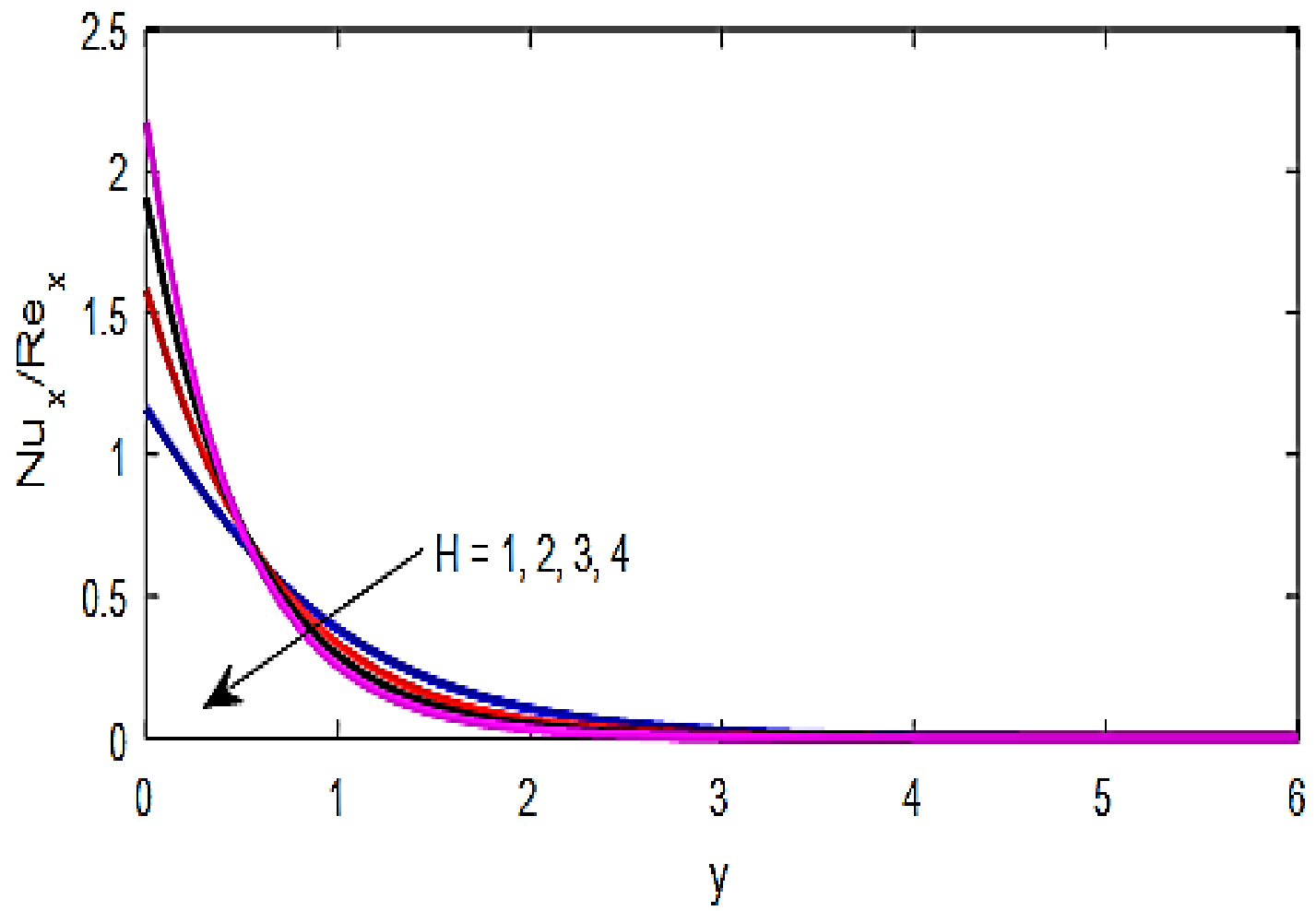

Figure 17: Variations of Nusselt number $N u / R e_{x}$ for different values of $H$ when $K=0.5$, $M=2, G r=10, G c=5, \alpha=\pi / 4, \beta=1, F=0.1, H_{1}=1, S c=2, K r=0.1, U_{p}=0.5$. 


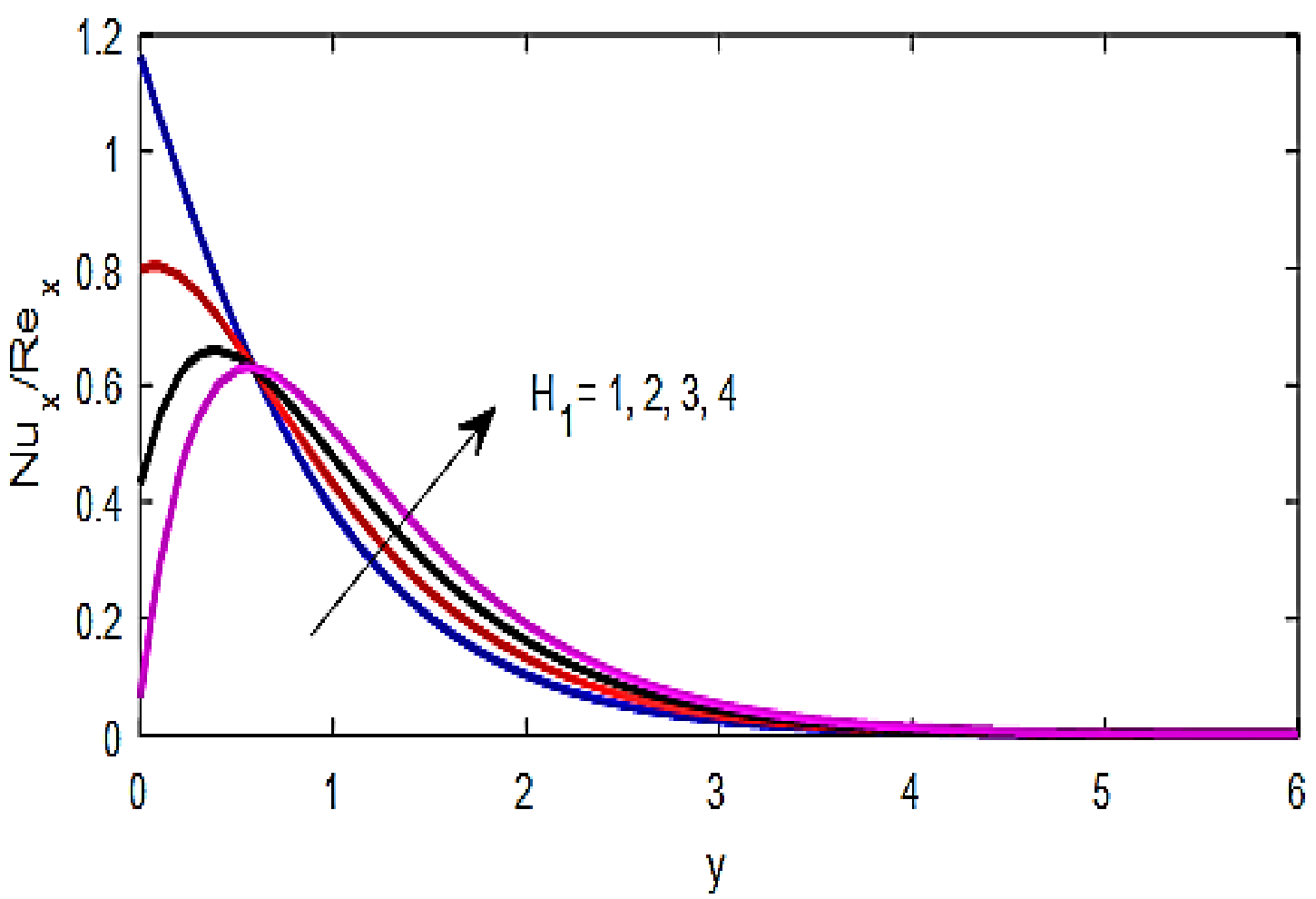

Figure 18: Nusselt number $N u / R e_{x}$ for different values of $H_{1}$ when $K=0.5, M=2$, $G r=10, G c=5, \alpha=\pi / 4, \beta=1, F=0.1, H=1, S c=2, K r=0.1, U_{p}=0.5$.

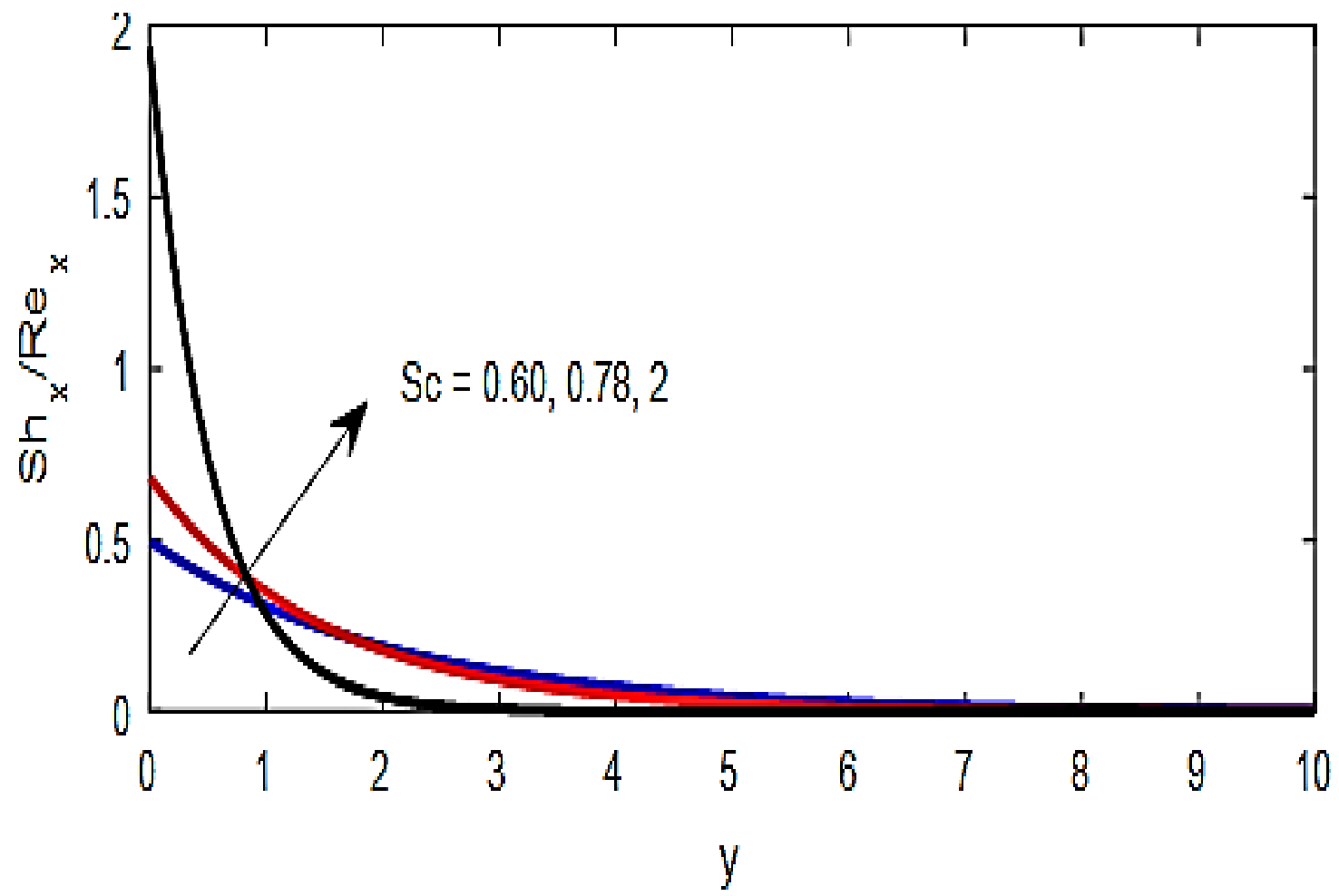

Figure 19: Sherwood number $S h / \operatorname{Re}_{x}$ for different values of $S c$ when $K=0.5, M=2$, $G r=10, G c=5, \alpha=\pi / 4, \beta=1, F=0.1, H=1, H_{1}=1, K r=0.1, U_{p}=0.5$. 
Figure 2 illustrates the velocity profiles for different values of thermal Grashof number $G r$. This parameter describes the relative magnitude of the buoyancy force and viscous forces acting on the micropolar fluid. Grashof number $G r>0$ for cooling, $G r<0$ for heating and $G r=0$ implies an absence of free convection currents. The velocity magnitudes are evidently enhanced for an inclined plate with an increase in thermal Grashof number. Momentum boundary layer thickness is therefore reduced. This is attributable to the dominance of buoyancy forces over the viscous forces, which serves to assist momentum diffusion and accelerates the flow. Furthermore, it is also noticed that the fluid velocity magnitude commences with the plate velocity, increases with distance from the surface, attains a maximum value near the plate and thereafter decreases monotonically to zero at the free stream. Figure 3 presents the response in linear velocity and micro-rotation to a variation in species (solutal) Grashof number $G c$.This parameter quantifies the relative contribution of species buoyancy force to viscous hydrodynamic force. With increasing $G c$, the exacerbation in mass diffusion leads to an acceleration in the flow i.e. increase in velocity values and an associated decrease in hydrodynamic boundary layer thickness. We note that for the case $G m=0$, species buoyancy effect vanishes and the momentum equation (19) is de-coupled from the species diffusion (concentration) equation (22).

Figure 4 shows the influence of angle of inclination $\alpha$ of the surface on velocity profiles. It is clearly observed that velocity is decreased with an increase of angle of inclination. This is attributable to the greater drag experienced at the plate surface relative to the decrease in thermal and species buoyancy forces. Greater effort is therefore needed to drive the micropolar fluid along the plate. Furthermore, the buoyancy effects decrease to a component of the maximum buoyancy force for a vertical plate, since the buoyancy forces scale with the factor $\cos \alpha$. Hence the fluid attains high velocity profiles for the vertical plate (i.e., $\alpha=\mathrm{O}^{\circ}$ ) and progressively decreases with greater inclination of the plate.

Figure 5 shows the pattern of the velocity for different values of magnetic field parameter $M$ It is observed that the amplitude of the velocity is reduced and momentum boundary layer thickness increases when $M$ is increased. Physically, in magnetohydrodynamic materials processing, the applied magnetic field exerts a retarding effect on the free convective flow, transverse to the direction of imposition of the magnetic field. With increasing the values of $M$, this type of resisting force slows down the fluid i.e. with stronger magnetic field strength the flow is decelerated and this is confirmed with the decreasing velocity distribution across the boundary layer. Furthermore, an asymptotically smooth solution is obtained for high values of the transverse coordinate $(y)$ indicating that a 
sufficiently large infinity boundary condition is prescribed in the free stream in the numerical computations. Linear momentum boundary layer thickness is therefore increased with greater magnetic parameter.

Figure 6 shows the graphical representation of the non-dimensional velocity on Eringen micropolar vortex viscosity parameter $\beta$. It is seen that as $\beta$ increases, the velocity gradient near the porous plate decreases, and then approaches to the free stream velocity. Comparison of the velocities for the permeability $K=5$ and $K=0.5$ cases indicates that, the velocity is greater as permeability increases than that of lesser values of permeability at the same values of $\beta$. The acceleration in the fluid with higher permeability is caused by the decrease in Darcian drag force. Also, it is noteworthy that velocity distribution across the boundary layer is lower for Newtonian fluid ( $\beta=0)$ as compared with stronger micropolar fluid $(\beta=2)$ for the same conditions and fluid properties. Micropolarity (i.e. increasing vortex viscosity of micro-elements) therefore consistently induces deceleration in the flow adjacent to the plate. All profiles are parabolic and peak at some distance from the wall, decaying smoothly to vanish in the free stream. A sufficiently larger infinity boundary condition is again confirmed in the profiles.

Figure 7 represents the influence of the plate moving velocity $U_{p}$ on velocity the direction of the fluid flow. It is noticed that the peak value of velocity across the boundary layer increases near the porous plate as the plate velocity increases. The linear flow is therefore accelerated with greater plate velocity. The translation of the plate induces a momentum boost in the flow. This pulls the boundary layer faster with greater $U_{p}$ values leading to a decrease in momentum boundary layer thickness.

Figure 8 visualizes the effect of micro-rotation vector component $n_{1}$ on microrotational (angular velocity) and permeability parameter $(K)$. It is apparent that micro-rotational velocity becomes increasingly negative for higher values of permeability parameter $K$ due to increase in the porosity of the porous medium. The greater permeability encourages reverse spin of micro-elements in the regime. Since the parameter $n_{1}$ is associated with the boundary condition equation (23), and also corresponds to the vanishing of the anti-symmetric part of the stress tensor, it is related to the weak concentration of the microelement of micropolar fluid. As $n_{1}$ increases, this encourages a reversal in spin of the micro-elements leading to larger negative values of the angular velocity. This trend has also been computed by many other investigators including Takhar et al. [50]. 
Figure 9, presents the effect of the thermal radiation-conduction parameter $F$ on temperature. This parameter defines the relative contribution of thermal radiation heat transfer to thermal conduction heat transfer. When $F>1$ thermal radiation dominates over thermal conduction, for $F<1$ thermal conduction dominates. When $F=1$ both thermal conduction and thermal radiation contributions are equal. For the present simulations, we confine attention to the case of $F<1$. Increasing radiation-conduction parameter is found to increase temperatures in the boundary layer. Thermal boundary layer thickness is therefore also enhanced with greater values of $F$. The boundary layer is energized with greater radiative flux contribution which leads to higher temperatures.

Figure 10 depicts the influence of heat source parameter, $H$ on temperature distribution in the flow. The heat absorption parameter $H$ appearing in (21) quantifies the amount of heat absorbed per unit volume which is given by $Q^{\prime}\left(T_{w}^{\prime}-T_{\infty}^{\prime}\right), Q^{\prime}$ being a constant coefficient, which may be taken as either positive or negative. The source term represents heat absorption for $H>0$ and heat generation when $H<0$.Physically speaking, the presence of heat absorption has the tendency to reduce the fluid temperature. Greater heat absorption $H$ clearly reduces the temperatures in the domain and the effect is most prominent at the wall. Heat sources and sinks may therefore be utilized to great effect in materials processing systems and indeed can be introduced relatively easily in porous media.

Figure 11 presents the evolution in temperature profiles with variation in radiation absorption parameter $H_{1}$. It shows that temperature increases as radiation absorption parameter increases. Physically speaking when heat is absorbed the buoyancy force tends to accelerate the flow. Thermal boundary layer thickness for micropolar fluids is therefore greater than other fluids. The large values of $H_{1}$ corresponds to an increased dominance of conduction over radiation absorption which manifests in an enhancement in buoyancy force and thickness of the thermal boundary layer.

Figure 12 displays the effect of Eckert number Ec on temperature profiles. This parameter is associated with the viscous heating effect. It signifies the quantity of mechanical energy converted via internal friction to thermal energy i.e. heat dissipation. It therefore represents the quantity of conversion of kinetic energy into internal energy by work done against the viscous fluid stresses. An increase in Eckert number via dissipation of mechanical energy into thermal energy will enhance the temperature of the micropolar fluid in the porous regime. Positive Eckert number implies cooling of the wall and therefore a transfer of heat to the micropolar fluid. Convection is therefore 
intensified and temperatures are markedly increased with greater Eckert number. Very smooth decays in temperature profiles are observed for all values of Eckert number and the convergence of profiles in the free stream once again confirms that an adequately large infinity boundary condition has been imposed in the present numerical method.

Figure 13 presents the evolution in dimensionless concentration profiles with variation in Schmidt number $S c$, which relates the thickness of the hydrodynamic boundary layer to that of the concentration boundary layer. The Schmidt number embodies the ratio of the momentum to the mass diffusivity i.e. $S c=v / D$.It is observed from the figure that the concentration profiles are decreased as the Schmidt number $S c$ is increased. As expected, mass transfer rate increases as $y$ increases, for all other parameters fixed, i.e. an increase in $S c$ decreases the concentration boundary layer thickness which is associated with the reduction in the concentration profiles.

Figure 14, illustrates the evolution in concentration with a change in chemical reaction parameter, $K r$. The reaction parameter is based on $a$ first-order irreversible chemical reaction which takes place both in the bulk of the fluid (homogeneous) as well as at plate which is assumed to be catalytic to chemical reaction. In the present study, we consider the non-destructive i.e. generative ( $K r>0)$ type of homogenous chemical reaction. It is noticed that concentration is enhanced in the boundary layer with greater chemical reaction, since more species is produced via the chemical reaction. This results in an enhancement in the thickness of the concentration boundary layer. These trends for the chemically reacting micropolar fluid concur closely with other studies including, for example, Modather et al. [51].

Figures 15-19 present the influence of various parameters on skin friction $C_{f}$, the couple stress, $C_{w}$, the Nusselt number i.e. wall temperature gradient $N u / R e_{X}$ and local Sherwood number i.e. wall temperature concentration gradient $S h / R e_{x}$. Figure 15 shows the effect of magnetic parameter on skin friction in the presence of other pertinent parameters characterizing the flow phenomena. It is interesting to observe that an increase in magnetic parameter skin friction coefficient reduces within the domain $y \leq 0.8$ (approx..). However, the reverse effect is encountered for $y>0.8$ i.e. skin friction increases with magnetic parameter. The two-layer variation in the profiles remarked due to the combination of various values of parameters which cause a deviation further from the plate in the customary behaviour of skin friction i.e. reduction with magnetic parameter. However, the dominant influence at and closer to the plate surface is a strong deceleration in the boundary layer flow with 
greater magnetic field. Figure 16 that the couple stress coefficient is decreased significantly with increasing values of magnetic parameter i.e. micro-rotation of micro-elements is stifled with greater transverse magnetic field (rotary motions are inhibited). Figure 17 exhibits the effect of heat absorption parameter, $H$ on the rate of heat transfer profiles and demonstrates that with increasing values of $H$ near the plate $y \leq 0.5$ the Nusselt number is enhanced whereas it is reduced further from the wall. Increasing radiation absorption parameter, $H_{1}$ results in a significant loss in the rate of heat transfer coefficient near the plate for which the boundary layer thickness decreases. Further, in the ambient state, Nusselt number increases with an increase in radiation absorption parameter (Figure 17). Figure 18 illustrates the effect of Schmidt number on rate of mass transfer coefficient i.e. Sherwood number for fixed values of other physical parameters prescribed. It is evident that the Sherwood number increases with an increase in Schmidt number. This implies that lighter species (higher molecular diffusivity) is favourable to enhance the rate of mass transfer.

\section{CONCLUSIONS}

A mathematical model has been developed for unsteady magnetohydrodynamic mixed convection heat and mass transfer of an oscillatory viscous incompressible, electrically- conducting, heat absorbing and radiating micropolar fluid flow from an inclined porous plate in a porous medium. The model includes chemical reaction, heat source, radiation absorption and Joule dissipation effects. Numerical solutions are obtained based on a perturbation analysis of the transformed conservation equations. A parametric study of the emerging parameters on nondimensional velocity, angular velocity, temperature and concentration profiles. Validation of the solutions with previous perturbation solutions derived by Pal and Biswas [26] is conducted. Excellent correlation is achieved. The main findings of the present investigation may be summarized as follows

- It is found that, the velocity is decreased with an increase in magnetic parameter, angle of inclination and Eringen micropolar vortex viscosity parameters. The reverse trend is observed with increasing Grashof number, modified Grashof number and plate moving velocity.

- Micro-rotation (angular velocity) decreases as micro-gyration parameter increases for higher values of permeability.

- An increase in the thermal radiation, radiation absorption parameter and Eckert number leads to an enhancement in temperature and thermal boundary layer thickness. The opposite behaviour is computed with increasing heat absorption parameter. 
- Species concentration decreases with increasing values of Schmidt number and positive values of chemical reaction parameter (generative reaction) whereas it is reduced with negative values of reaction parameter (destructive reaction).

- Generally skin friction is strongly reduced with increasing magnetic field.

- Couple stress coefficient is reduced significantly with increasing values of magnetic parameter.

- Sherwood number (rate of mass transfer coefficient) increases with an increase in Schmidt number.

The study is relevant to multi-physical modelling of magnetic polymer processing. Future studies will consider more complex fluid such as nanofluid with different bases and will be communicated soon.

\section{REFERENCES}

[1] Bhaumik, S.K, Behera, R, CFD optimization of free convective cooling of finned heat sink: Effect of fin spacing, Procedia Eng, 127, 155-161 (2015). doi: 10.1016/j.proeng.201.11.318

[2] Khedr, M.E.M, Chamkha, A.J, Bayomi, M, MHD flow of a micropolar fluid past a stretched permeable surface with heat generation or absorption, Nonlinear Analysis, Modelling and Controlling, 14(1), 27-40 (2009).

[3] Pal, D, Biswas, S, Influence of Heat sink on Magento-thermal radiative convective oscillatory flow of micropolar fluid in a porous medium with convective boundary condition, Int. J. Appl. Comput. Math, (2016). doi: 10.1007/540819-016-0211-6

[4] Srinivasa Raju, R, Jithender Reddy, G, Anand Rao, J, Rashidi, M.M, Gorla, R.S.R, Analytical and numerical study of unsteady MHD free convection with heat absorption, Int. J. Ther. Sci, 107, 303-315 (2016).

[5] Mishra, S.R, Dash, G.C, Pattnaik, P.K, Flow of heat and mass transfer on MHD free convection in a micropolar fluid with heat source, Alexandria. Eng. J, 54(3), 681-689 (2015).

[6] Alam, S, Islam, T, Uddin, M.J, Mathematical modelling for heat transfer of a micropolar fluid along a permeable stretching/shrinking wedge with heat generation/absorption, Math. Modelling Eng. Prob, 3(1),1-9 (2016). doi: 10.18280/mmep.030101

[7] Mishra, S.R, Dash, G.C, Acharya, M, Mass and heat transfer effects on MHD flow of a viscoelastic fluid through porous medium with oscillatory suction and heat source, Int. J. Heat and Mass Transfer, 57(2), 433438 (2013)

[8] Tripathy, R.S, Mishra, S.R, Dash, G.C, Hoque, M.M, Numerical analysis of hydromagnetic micropolar fluid along a stretching sheet with non- uniform heat source and chemical reaction, Eng. Sci. Tech. An Int. J, 19,1573-1581 (2016)

[9] Dombrovsky, L.A, Sazhin, S.S, Absorption of thermal radiation in semi-transparent spherical droplet: A simplified model, Int. J. Heat Fluid Flow, 24, 919-927 (2003)

[10] Shercliff, J.A, A Text Book of Magnetohydrodynamics, Pergamon Press, New York, USA (1965)

[11] Satyanarayana, P.V, Venkateswarlu. B, Venkata Ramana. S, Effect of Hall current and Radiation absorption on MHD micropolar fluid in a rotating system, Ain Shams Eng. J, 4, 843-854 (2013)

[12] Kundu, P.K, Das, K, Jana, S, MHD micropolar fluid flow with thermal radiation and thermal diffusion in a rotating frame, Bull. Malays. Math. Sci. Soc, 38, 1185-1205 (2015). doi: 10.1007/s40840-014-0061-5 
[13] Harish Babu, D, Satyanarayana, P.V, Influence of variable permeability and Radiation absorption on heat and mass transfer in MHD micropolar flow over a vertical moving porous plate, ISRN Thermodynamics, ArticleID:953536 (17pages) (2013). http://dx.doi.org/10.1155/2013/953536

[14] Gebhart, B, Effect of viscous dissipation in natural convection, J. Fluid Mech, 14, 225-232 (1962).

[15] Rahman, M.M, Convective flows of micropolar fluids from radiative isothermal porous surfaces with viscous dissipation and Joule heating, Commun. Nonlinear. Sci. Simulat, 14, 3018-3030 (2009).

[16] Ziaul Haque, M.D, Mahmud Alam, M.D, Ferdows, M, Postelnicu, A, Micropolar fluid behaviours on steady MHD free convection and mass transfer flow with constant heat and mass fluxes, Joule heating and viscous dissipation, J. King Saud Uni.-Eng. Sci, 24, 71-84 (2012).

[17] Reddy, M.G, Padma, P, Shanker, B, Effects of viscous dissipation and heat source on unsteady MHD flow over a stretching sheet, Ain Shams Eng. J, 6, 1195-1201 (2015).

[18] Reddy, M.G, Gorla, R.S.R, Micropolar fluid flow over a nonlinear stretching convectively heated vertical surface in the presence of Cattaneo-Christov heat flux and viscous dissipation, Frontiers in Heat and Mass Transfer, 8, 003020 (2017) doi: 10.5098/hmt.8.20.

[19] Reddy, M.G, Influence of Lorentz force, Cattaneo-Christov heat flux and viscous dissipation on the flow of micropolar fluid past a nonlinear convective stretching vertical surface, Nonlinear Eng, (2017). https://doi.org/10.1515/nleng-2017-0043.

[20] Siva Reddy, S, Shamshuddin, M.D, Heat and mass transfer on the MHD flow of a micropolar fluid in the presence of viscous dissipation and chemical reaction, Procedia Eng, 127, 885-892 (2015).

[21] Rout, P.K, Sahoo, S.N, Dash, G.C, Mishra, S.R, Chemical reaction effect on MHD free convection flow in a micropolar fluid, Alexandria. Eng. J, (2016). http://dx.doi.org/10.1016/j.aej.2016.04.033.

[22] Pal, D, Talukdar, B, Perturbation technique for unsteady MHD mixed convection periodic flow, heat and mass transfer in micropolar fluid with chemical reaction in the presence of thermal radiation, Central European $J$. Phys, 10, 1150-1167 (2012).

[23] Reddy, M.G, Thermal Radiation and Chemical Reaction Effects on Steady Convective Slip Flow with Uniform Heat and Mass Flux in the Presence of Ohmic Heating and a Heat Source, Fluid Dynamics \& Materials Processing, 10(4), 417-442 (2015).

[24] Reddy, M.G, Heat and mass transfer on magnetohydrodynamic peristaltic flow in a porous medium with partial slip, Alexandria Eng. J, 55, 1225-1234 (2016).

[25] Bég, O.A, Bég, T.A, Bakier, A.Y, Prasad, V, Chemically-reacting mixed convective heat and mass transfer along inclined and vertical plates with Soret and Dufour effects: Numerical solutions, Int. J. Appl. Math. Mech, 5(2), 39-57 (2009).

[26] Pal, D, Biswas, S, Perturbation analysis of magnetohydrodynamics oscillatory flow on convective-radiative heat and mass transfer of micropolar fluid in a porous medium with chemical reaction., Eng. Sci. Tech: An Int. $J, 19,444-462$ (2016).

[27] Rahman, M.M, Aziz, A, Al-Lawatia, M.A, Heat transfer in micropolar fluid along an inclined permeable plate with variable fluid properties, Int. J. Ther. Sci, 49(6), 993-1002 (2010).

[28] Ajaz Ahmad, D, Elangovan, K, Effect of inclined magnetic field on the oscillatory flow of micropolar fluid in a porous micro-channel under the action of alternating electric field, World J. Eng. Tech, 2(6), 125-145 (2016).

[29] Aurangzaib, Kasim, A.R.M, Mohammad, N.F, Shafie, S, Unsteady MHD mixed convection flow of a micropolar fluid along an inclined stretching plate, Heat Transfer Asian Research, 42, 89-99 (2013). 
[30] Srinivasacharya, D, Himabindu, K, Entropy generation in a micropolar fluid flow through an inclined channel, Alexandria Eng. J, 55, 973-982 (2016).

[31] Bég, O.A, Zueco, J, Chang, T.B, Numerical analysis of hydromagnetic gravity-driven thin film micropolar flow along an inclined plane, Chemical Eng. Commun, 198(3), 312- 331 (2015).

[32] Bég, O.A, Ghosh, S.K, Narahari, M, Bég, T.A, Mathematical modelling of thermal radiation effects on transient gravity-driven optically-thick gray convection flow along an inclined plate with pressure gradient, Chemical Eng. Commun, 198, 1-15 (2011).

[33] Bég, O.A, Ghosh, S.K, Ahmed, S, Bég, T.A, Mathematical modelling of oscillatory magneto-convection of a couple stress biofluid in an inclined rotating channel, J. Mechanics in Medicine and Biology, 12(3), 12500501 to $1250050-35$ (2012).

[34] Rana, P, Bhargava, R, Bég, O.A, Numerical solution for mixed convection boundary layer flow of a nanofluid along an inclined plate embedded in a porous medium, Computers and Mathematics with Applications, 64, 2816-2832 (2012).

[35] Rao, A.S, Prasad, V.R, Nagendra, N, Reddy, N.B, Bég, O.A, Non-similar computational solution for boundary layer flows of non-Newtonian fluid from an inclined plate with thermal slip, J Appl. Fluid Mech, 9(2), 795807 (2016).

[36] Douglas, E.P, Processing of polymers using magnetic fields. Proc. International Workshop on Materials Analysis and Processing in Magnetic Fields, Tallahassee, Florida (2004) March:17 - 19.

[37] Eringen, A.C, Theory of micropolar fluids, J. Appl. Math. Mech, 16, 1-18 (1966).

[38] Bég, O.A, Motsa, S.S, Kadir, A, Bég, T.A, Islam, M.N, Spectral quasilinear numerical simulation of micropolar convective wall plumes in high permeability porous media, J. Eng. Thermophys, 25(4), 1-24 (2016).

[39] Bhargava, R, Sharma, S, Bhargava, P, Bég, O.A, Kadir, A, Finite element simulation of nonlinear convective heat and mass transfer in a micropolar fluid-filled enclosure with Rayleigh number effects, Int. J. Appl. Comput. Math, (2016). doi:10.1007/s40819-016-0180-9.

[40] Eringen, A.C, Theory of thermo-micropolar fluids, J. Math. Appl, 38, 480-495 (1972).

[41] Mishra, S.R, Jena, S, Numerical solution of boundary layer MHD flow with viscous dissipation, The Scientific World Journal, Article ID: 756498 (2014). doi:10.1155/2014/756498

[42] Guram, G.S, Smith, A.C, Stagnation flows of micropolar fluids with strong and weak interactions, Computers and Mathematics with Applications, 6, 231-233 (1980).

[43] Ahmadi, G, Self-similar solution of incompressible micropolar boundary layer flow over a semi-infinite plate, Int. J. Eng. Sci, 14, 639-646 (1976).

[44] Peddieson, J, An application of the micropolar fluid model to the calculation of turbulent shear flow, Int. J. Eng. Sci, 10, 23-32 (1972).

[45] Stokes, V.K, Theories of fluids with Microstructure, Springer, New York (1984).

[46] Adunson, T, Gebhart. B, An experimental and analytical study of natural convection with appreciable thermal radiation effects, J. Fluid Mech, 52, 57-95 (1972).

[47] Uddin, M.J, Alginahi, Y, Bég, O.A, Kabir, M.N, Numerical solutions for nonlinear gyrotactic bio convection in nanofluid saturated porous media with Stefan blowing and multiple slip effects, Computers and Mathematics with Applications, 72(10), 2562-2581 (2016). 
[48] Bég, O.A, Basir, M.F.M, Uddin, M.J, Ismail, A.I.M, Numerical study of slip effects on asymmetric bio convective nanofluid flow in a porous microchannel with an expanding/contracting upper wall using Buongiorno's model, J. Mechanics in Medicine and Biology, (2016). doi.:10.1142/S0219519417500592.

[49] Reddy, M.G, Sandeep, N, Free convective heat and mass transfer of magnetic bio-convective flow caused by a rotating cone and plate in the presence of nonlinear thermal radiation and cross diffusion, J. Comput. Appl. Res. Mech. Eng, 7(1), 1-21 (2017).

[50] Takhar, H, Agarwal, R, Bhargava, R, Mixed convection flow of a micropolar fluid over a stretching sheet, Heat and Mass Transfer, 34, 213-219 (1998).

[51] Modather, M, Rashad, A.M, Chamkha, A.J, An analytical study of MHD heat and mass transfer oscillatory flow of a micropolar fluid over a vertical permeable plate in porous medium, Turk. J. Eng. Env. Sci, 33(4), 245-257 (2009).

\section{APPENDIX}

The set of coupled nonlinear governing boundary-layer equations (20) - (27) together with the boundary conditions, equation (28) are solved numerically using Runge-Kutta method along with shooting technique. The higher order nonlinear differential equations (20) - (27) are converted into simultaneous nonlinear differential equations of first order and they are further transformed into initial value problem by applying the shooting technique. The resultant initial value problem is solved by employing Runge-Kutta fourth order method. The step size $\eta=0.001$ is used to obtain the numerical solution. From the process of numerical computation, the skinfriction coefficient, the couple stress coefficient, the Nusselt number and Sherwood number which are respectively proportional to $u^{\prime}(0), w^{\prime}(0),-\theta^{\prime}(0)$ and $-\phi^{\prime}(0)$ are also sorted out and then presented in graphs.

The numerical procedure as follows:

Let,

$u_{0}=y_{1}, u_{0}^{\prime}=y_{2}, u_{1}=y_{3}, u_{1}^{\prime}=y_{4}, \omega_{0}=y_{5}, \omega_{0}^{\prime}=y_{6}, \omega_{1}=y_{7}, \omega_{1}^{\prime}=y_{8}$,

$\theta_{0}=y_{9}, \theta_{0}^{\prime}=y_{10}, \theta_{1}=y_{11}, \theta_{1}^{\prime}=y_{12}, \phi_{0}=y_{13}, \phi_{0}^{\prime}=y_{14}, \phi_{1}=y_{15}, \phi_{1}^{\prime}=y_{16}$

Hence,

\section{Zeroth order}

$$
\begin{aligned}
& \frac{\partial^{2} u_{0}}{\partial y^{2}}=y_{2}^{\prime}=-\frac{1}{1+\beta}\left(y_{2}+G r y_{9} \cos \alpha+G c y_{13} \cos \alpha-\left(M+\frac{(1+\beta)}{K}\right) y_{1}+2 \beta y_{6}\right) \\
& \frac{\partial^{2} \omega_{0}}{\partial y^{2}}=y_{6}^{\prime}=-\eta y_{6} \\
& \frac{\partial^{2} \theta_{0}}{\partial y^{2}}=y_{10}^{\prime}=-\frac{P r}{(1+F)}\left(y_{10}-\frac{H}{P r} y_{9}+H_{1} y_{13}+E c\left(y_{2}^{2}+M y_{1}^{2}\right)\right) \\
& \frac{\partial^{2} \phi_{0}}{\partial y^{2}}=y_{14}^{\prime}=-S c\left(y_{14}+K r y_{13}\right)
\end{aligned}
$$




\section{First order}

$\frac{\partial^{2} u_{1}}{\partial y^{2}}=y_{4}^{\prime}=-\frac{1}{1+\beta}\left(y_{3}+G r y_{11} \cos \alpha+G c y_{15} \cos \alpha-\left(M+\frac{(1+\beta)}{K}\right) y_{1}+A y_{2}+2 \beta y_{8}\right)$

$\frac{\partial^{2} \omega_{1}}{\partial y^{2}}=y_{8}^{\prime}=-\left(\eta y_{8}-n \eta y_{7}+A \eta y_{6}\right)$

$\frac{\partial^{2} \theta_{1}}{\partial y^{2}}=y_{12}^{\prime}=-\frac{P r}{(1+F)}\left(y_{12}-\frac{H}{P r} y_{11}-n y_{11}+H_{1} y_{15}+A y_{10}+E c\left(2 y_{2} y_{4}+2 M y_{1} y_{3}\right)\right)$

$\frac{\partial^{2} \phi_{1}}{\partial y^{2}}=y_{16}^{\prime}=-S c\left(y_{16}+(K r-n) y_{15}+A \eta y_{14}\right)$

with the boundary conditions

$y_{a}(1)=U_{p}, y_{a}(3)=0, y_{a}(5)=-n_{1} y_{a}(2), y_{a}(7)=-n_{1} y_{a}(4), y_{a}(9)=1, y_{a}(11)=1, y_{a}(13)=1, y_{a}(15)=1$,

$y_{b}(1)=0, y_{b}(3)=0, y_{b}(5)=0, y_{b}(7)=0, y_{b}(9)=0, y_{b}(11)=0, y_{b}(13)=0, y_{b}(15)=0$

Where $a$ and $b$ are used for initial and boundary conditions respectively.

\section{NOMENCLATURE}

\section{Roman}

A small real positive constant

$B_{0} \quad$ Magnetic field strength

C Concentration of the solute $\left[\mathrm{mol} \mathrm{m}^{-3}\right]$

$C_{f} \quad$ Skin friction coefficient

$C_{w} \quad$ Wall couple stress

$C_{p} \quad$ Specific heat at constant pressure $\left[\mathrm{J} \mathrm{Kg}^{-1} \mathrm{~K}^{-1}\right]$

$C_{\infty} \quad$ Free stream concentration $\left[\mathrm{mol} \mathrm{m}^{-3}\right]$

$D_{m} \quad$ Molecular diffusivity $\left[\mathrm{m}^{2} \mathrm{~s}^{-1}\right]$

Ec Eckert number

$F \quad$ radiation conduction parameter

$g \quad$ Acceleration due to gravity $\left[\mathrm{ms}^{-1}\right]$

$G_{m} \quad$ Solutal Grashof number

$G_{r} \quad$ Grashof number 
$H \quad$ Heat absorption parameter

$H_{1} \quad$ radiation absorption parameter

$j^{\prime} \quad$ micro inertia per unit mass $\left[\mathrm{m}^{2}\right]$

$j \quad$ dimensionless micro inertia per unit mass

$K^{\prime} \quad$ permeability parameter $\left[\mathrm{m}^{2}\right]$

$K \quad$ dimensionless permeability parameter

$\mathrm{Kr} \quad$ chemical reaction parameter

M magnetic field parameter

$n \quad$ frequency parameter [ hertz]

$n_{1} \quad$ parameter related micro-gyration vector and shear stress

$\mathrm{Nu} \quad$ Nusselt number

$p \quad$ constant pressure

$\operatorname{Pr} \quad$ Prandtl number

$q_{r} \quad$ Radiative heat flux $\left[\mathrm{Wm}^{-2}\right]$

$R e_{x}$ local Reynolds number

Sc Schmidt number

Sh Sherwood number

$t^{\prime} \quad$ dimensional time $[s]$

$t \quad$ dimensionless time

$T \quad$ Temperature of the field in the boundary layer [ $K$ ]

$T_{w} \quad$ wall temperature of the fluid [ $K$ ]

$T_{\infty} \quad$ Temperature of the fluid in free stream $[K]$

$u^{\prime} \quad$ velocity component in x-direction $\left[\mathrm{ms}^{-1}\right]$

$u \quad$ dimensionless velocity component in $\mathrm{x}$-direction 
$U_{0} \quad$ Free stream velocity $\left[m s^{-1}\right]$

$u_{p}^{\prime} \quad$ uniform velocity of the fluid in its own plane $\left[\mathrm{ms}^{-1}\right]$

$U_{p} \quad$ dimensionless velocity of the plate

$V_{0} \quad$ scale suction velocity at the plate $\left[\mathrm{ms}^{-1}\right]$

$v^{\prime} \quad$ velocity component in y-direction $\left[\mathrm{ms}^{-1}\right]$

$v \quad$ dimensionless velocity component in y-direction

$x, y$ distance along and perpendicular to the plate $[\mathrm{m}]$

\section{Greek symbols}

$\alpha$ angle of inclination

$\beta \quad$ viscosity ratio parameter

$\beta_{c} \quad$ volumetric coefficient of concentration expansion $\left[K^{-1}\right]$

$\beta_{f} \quad$ volumetric coefficient of concentration expansion $\left[K^{-1}\right]$

$\eta \quad$ dimensionless gyro-viscosity micropolar related parameter

$\gamma \quad$ spin gradient viscosity [ $\left.k g \mathrm{~ms}^{-1}\right]$

$\varepsilon \quad$ small positive quantity

$\theta \quad$ dimensionless temperature

$\mu \quad$ fluid dynamic viscosity

$v \quad$ kinematic viscosity $\left[m^{2} s^{-1}\right]$

$v_{r} \quad$ kinematic rotational viscosity $\left[\mathrm{m}^{2} \mathrm{~s}^{-1}\right]$

$\rho \quad$ density of micropolar fluid $\left[\mathrm{kg} \mathrm{m}^{-3}\right]$

$\sigma \quad$ electrical conductivity of the fluid $\left[S \mathrm{~m}^{-1}\right]$ 


$$
\begin{array}{ll}
\bar{\sigma} & \text { Stefan-Boltzmann constant }\left[\mathrm{Wm}^{-2} \mathrm{~K}^{-4}\right] \\
\kappa & \text { thermal conductivity }\left[\mathrm{Wm}^{-1} \mathrm{~K}^{-1}\right] \\
\bar{\kappa} & \text { mean absorption coefficient }\left[\mathrm{m}^{-1}\right] \\
\omega^{\prime} & \text { component of angular velocity }\left[\mathrm{m}^{2} \mathrm{~s}^{-2}\right] \\
\omega & \text { dimensionless angular velocity component } \\
\Lambda & \text { coefficient of gryo-viscosity }\left[\mathrm{kg} \mathrm{ms}^{-1}\right] \\
\phi & \text { dimensionless concentration }
\end{array}
$$

\title{
Subscripts
}

\author{
$w \quad$ surface conditions \\ $\infty$ conditions far away from the plate
}

